\title{
Impact de l'éolien sur le réseau de transport et la qualité de l'énergie
}

\author{
B. Robyns ${ }^{1,2}$, A. Davigny ${ }^{1,2}$, C. Saudemont ${ }^{1,2}$, A. Ansel $^{1,2}$, V. Courtecuisse ${ }^{1,2}$ \\ B. François ${ }^{1,3}$, S. Plumel ${ }^{4}$, J. Deuse ${ }^{5}$ \\ Centre National de Recherche Technologique (CNRT FUTURELEC) \\ Laboratoire d'Electrotechnique et d'Electronique de Puissance de Lille (L2EP) ${ }^{1}$ \\ Ecole des Hautes Etudes d'Ingénieur (HEI), 13 rue de Toul, F-59046 Lille Cedex, France ${ }^{2}$ \\ Ecole Centrale de Lille, Cité Scientifique, BP 48, 59651 Villeneuve d'Ascq Cedex, France ${ }^{3}$ \\ Supélec, Plateau de Moulon, 3 rue Joliot - Curie, 91192 Gif - sur - Yvette cedex, France ${ }^{4}$ \\ Suez - Tractebel, avenue Ariane 7, B-1200 Bruxelles, Belgique ${ }^{5}$
}

\begin{abstract}
Résumé--L'augmentation de la puissance éolienne installée en Europe a un impact croissant sur le réseau de transport du fait de la difficulté à prévoir la production, de la capacité d'accueil limitée du réseau, du risque de déconnexions intempestives des fermes d'éoliennes et d'une dégradation de la qualité de l'électricité. Cet article propose une synthèse de cette problématique après avoir présenté les technologies d'éoliennes de grande puissance et les contraintes liées à leur raccordement au réseau de transport. Des relevés expérimentaux et des résultats de simulation illustrent la qualité de la puissance générée par différentes technologies d'éoliennes isolées ou en ferme, l'impact de ces éoliennes sur un réseau électrique et certaines possibilités de réglage qui permettraient d'améliorer leur intégration dans le réseau.
\end{abstract}

Mots - clés--Eolienne à vitesse fixe, Eolienne à vitesse variable, Machine Asynchrone à Double Alimentation, Réseau de transport, Services système.

\section{INTRODUCTION}

$\mathrm{S}$ elon l'EWEA (European Wind Energy Association) le

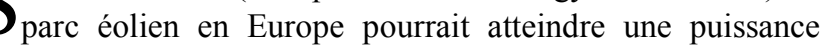
installée de $180000 \mathrm{MW}$ en 2020, soit plus de cinq fois la puissance installée en 2004 qui était de $34000 \mathrm{MW}$.

Pour satisfaire aux objectifs de développement des énergies renouvelables de l'Union Européenne, la puissance éolienne installée en France en 2010 devrait atteindre 14000 MW. Avec plus de $600 \mathrm{MW}$ installés en 2005, on peut affirmer que cet objectif ne sera pas atteint en 2010, mais il reste d'actualité à plus long terme. L'impact de l'éolien sur le réseau de transport est cependant à considérer à une échelle européenne. D'après l'UCTE (Union for the Co-ordination of the Transmission of Electricity) [1], la concentration d'énergie éolienne dans le nord de l'Allemagne produit déjà d'importants flux discontinus de puissance sur les réseaux de transport voisins, du Benelux et d'Europe Centrale. Dans le futur, le réseau de transport français risque de ne pas être épargné par ce phénomène : en effet d'ici 2010, 20000 MW de puissance éolienne pourrait être installée en Allemagne et plus de $10000 \mathrm{MW}$ en Espagne, s'ajoutant aux milliers de
MW qui seraient installés en France [2].

Les problèmes induits par l'intégration d'éoliennes dans le réseau électrique sont causés par :

- leur production aléatoire et difficilement prévisible ;

- une absence de réglage puissance - fréquence ;

- une participation au réglage de la tension limitée pour les éoliennes à vitesse variable, et aucune participation à ce réglage pour les éoliennes dont la génératrice est directement couplée au réseau ;

- une sensibilité élevée aux creux de tension et aux variations de fréquence pour certaines technologies ;

- une sensibilité importante aux variations rapides de la force du vent.

Les problèmes majeurs de l'éolien sont la grande variabilité de sa production et surtout la difficulté de prévoir cette production précisément plusieurs heures à l'avance. L'expérience allemande montre que des prévisions à $72 \mathrm{~h}$ sont impossibles, et que l'erreur sur des prévisions à $24 \mathrm{~h}$ est en moyenne de $10 \%$ de la capacité installée et peut atteindre parfois $50 \%$ [2], [3].

Se pose aussi la question d'une adéquation entre la consommation et la puissance éolienne disponible? En général, il y a plus de vent en hiver qu'en été, durant le jour que la nuit, ce qui correspond aux tendances de la consommation en France où le pic hivernal peut atteindre plus de $80000 \mathrm{MW}$, tandis que le pic de l'été peut atteindre plus de $50000 \mathrm{MW}$. Cependant, il apparaît que durant les périodes de grandes chaleurs ou de grands froids, la puissance éolienne est très faible, voire nulle [2]. La multiplicité des installations éoliennes sur un vaste territoire est néanmoins favorable à une corrélation entre la consommation et la production éolienne comme le montrent des études sur le foisonnement éolien [4]. Le foisonnement éolien est le phénomène statistique qui fait que, par la multiplication spatiale des installations éoliennes sur un territoire donné, la valeur minimale de la puissance injectée par l'ensemble est supérieure à la somme des puissances minimales de chacune d'entre-elles. Ainsi, la multiplication d'éoliennes (soumises à des vitesses de vent différentes) conduit globalement à un lissage de la puissance 
générée. Les études prospectives réalisées sur un parc de 10000 MW installé en France, réparti entre le nord et le sud du territoire, montre l'existence d'une contribution minimale de l'éolien, par exemple de $25 \%$ environ pendant les périodes de pointes hivernales avec un niveau de probabilité de $90 \%$, car il y a toujours du vent qui souffle quelque part [4]. Cette contribution de l'éolien pourrait être améliorée par une gestion couplée des ressources éoliennes et hydrauliques [5]. Néanmoins, cela conduirait à des révisions majeures du plan de sécurité du réseau électrique.

Le fait de ne pas participer aux services système (réglage de la tension, de la fréquence, démarrage en autonome ou blackstart, possibilité de fonctionner en îlotage,...) amène les éoliennes à se comporter comme des générateurs passifs du point de vue électrique [6]. Le réglage de la tension et de la fréquence est dès lors reporté sur les alternateurs classiques. Le taux de pénétration de l'éolien, c'est-à-dire la puissance que génèrent les éoliennes par rapport à la puissance totale consommée, doit alors être limité afin de pouvoir garantir la stabilité du réseau dans des conditions acceptables [7]. Certains retours d'expériences (par exemple au Danemark) indiquent que, pour des taux de pénétration supérieurs à 20 ou $30 \%$, des problèmes de stabilité peuvent apparaître [7]. Une solution évidente pour le gestionnaire du réseau consiste alors à «augmenter» les capacités classiques de réglage reposant sur des groupes rapides (Turbines à gaz, groupes diesels), ce qui va à l'encontre des motivations écologiques visant au développement de la production éolienne.

La grande sensibilité de l'éolien aux perturbations du réseau, tels que les creux de tension ou les variations de fréquence, entraîne souvent une déconnexion de la production lors d'incidents sur le réseau. Cette déconnexion peut aggraver un déséquilibre production - consommation et par effet domino accélérer l'avènement d'un incident majeur dans le réseau. La tendance actuelle est, dès lors, de demander à cette production de rester connectée au réseau lors de creux de tension et de variations de fréquence dépassant certaines limites déterminées par les gestionnaires de réseau [3].

Les modalités de raccordement au réseau électrique des installations éoliennes sont définies par des décrets et arrêtés. Les installations d'une puissance maximale de $12 \mathrm{MW}$ sont connectées au réseau de distribution, généralement de moyenne tension (HTA). Normalement les installations d'une puissance supérieure à $12 \mathrm{MW}$ sont connectées au réseau de transport (HTB). A l'origine, le réseau HTA était en principe conçu pour accueillir des consommateurs; l'intégration progressive de productions dans ce réseau peut conduire jusqu'à une inversion des flux de puissance au niveau des postes HTA-HTB. En fonction du taux de pénétration, ceci peut influencer avant tout la gestion de la tension, mais peut aussi conduire à des révisions du plan de protection et à des modifications de structure en raison de courants de courtcircuit trop élevés.

L'obligation de rachat par EDF de l'électricité produite par des installations éoliennes d'une puissance inférieure ou égale à 12 MW a favorisé le développement de l'éolien dans le réseau HTA. Néanmoins, la tendance pour l'avenir est le développement de fermes éoliennes terrestres ou off-shore d'une puissance supérieure à $12 \mathrm{MW}$, voire très supérieure (de l'ordre de la centaine de MW), qui seront donc connectées au réseau HTB.

Les prescriptions techniques relatives au raccordement au réseau de transport d'une installation de production d'énergie électrique sont précisées dans le décret [8] et l'arrêté [9]. Au sens du décret [8], la qualité de l'électricité se vérifie par le maintien dans les plages contractuelles, réglementaires ou normatives :

- des paramètres caractéristiques des ondes de tension et de courant du réseau électrique ;

- de la continuité et de la fiabilité de l'alimentation des utilisateurs.

Ce deuxième critère de continuité et de fiabilité dépend essentiellement de la disponibilité de la source amont, le vent, ce qui par nature pose évidemment problème. La qualité des ondes de tension et courant au point de connexion des éoliennes au réseau dépend fortement de la technologie de ces éoliennes. C'est pourquoi, sont rappelées dans cet article les trois technologies d'éoliennes de grande puissance actuellement mises en oeuvre, en soulignant les capacités de réglage offertes par chacune. La qualité de la puissance générée par ces différents types d'éolienne est illustrée au moyen de mesures réalisées sur des éoliennes réelles ou sur un banc d'essai de laboratoire.

Les tensions et contraintes de raccordement de l'éolien au réseau de transport sont ensuite évoquées en se focalisant sur les problèmes induits par l'intégration de ces éoliennes dans ce type de réseau.

Des résultats de simulation illustrent l'impact d'une ferme éolienne sur la fréquence et la tension du réseau, ainsi que l'effet positif dû au foisonnement de l'éolien. La possibilité pour une éolienne à vitesse variable de participer au réglage primaire de fréquence est également illustrée.

\section{TECHNOLOGIES D'EOLIENNES DE GRANDE PUISSANCE}

Le principe de conversion de l'énergie cinétique du vent en énergie électrique et la description détaillée des différents types d'aérogénérateurs sont présentés dans plusieurs références [3], [10]-[13]. Dans cette section, sont présentées brièvement les trois technologies d'éoliennes de grande puissance les plus couramment installées, en soulignant leurs capacités de réglage de la puissance générée.

La puissance mécanique qui peut être extraite du vent se détermine au moyen de l'expression suivante :

$$
P=\frac{1}{2} \cdot \rho \cdot S \cdot C_{p} \cdot v^{3}
$$

où $\rho$ est la masse volumique de l'air, $S$ la surface balayée par la turbine, $v$ la vitesse du vent et $C_{p}$, le coefficient de puissance. 
Ce coefficient, correspondant au rendement aérodynamique de la turbine, a une évolution non linéaire en fonction du ratio de vitesse, $\lambda$ (Fig. 1) :

$$
\lambda=\frac{R_{t} \cdot \Omega_{t}}{v}
$$

où $R_{t}$ est le rayon de la turbine et $\Omega_{t}$ la vitesse mécanique de la turbine.

La caractéristique du coefficient de puissance varie avec l'angle d'orientation des pales $(\beta)$. La relation (1) montre qu'une petite variation de la vitesse du vent induit une grande variation de la puissance générée.

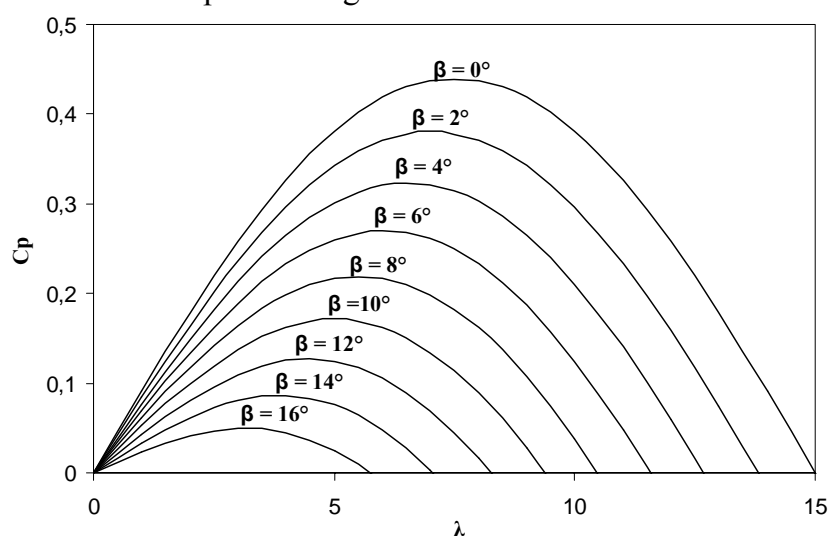

Fig. 1. Exemple d'évolution approchée du coefficient de puissance en fonction du ratio de vitesse $\lambda$ pour différents angles d'orientation des pales $\beta$.

\section{A. Eolienne à vitesse fixe}

Les premières éoliennes de grande puissance mises en œuvre reposent sur l'utilisation d'une machine asynchrone à cage directement couplée sur le réseau électrique (Fig. 2). Cette machine est entraînée par un multiplicateur et sa vitesse est maintenue approximativement constante par un système mécanique d'orientation des pales (pitch control).

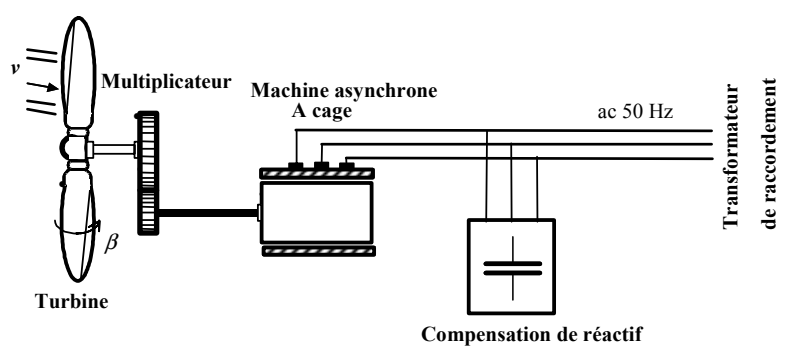

Fig. 2. Eolienne à vitesse fixe

La caractéristique de réglage théorique puissance-vitesse du vent est représentée en trait continu à la Fig. 3, pour une éolienne de $300 \mathrm{~kW}$. La dynamique relativement lente du pitch control (plusieurs dizaines de seconde) et les variations rapides de la vitesse du vent amènent ce type d'éolienne à suivre approximativement la caractéristique de réglage, comme illustré par les points reportés sur la Fig. 3, mesurés sur une éolienne réelle (de l'ancien site éolien de Dunkerque). La Fig.4 montre un enregistrement de la puissance générée par cette éolienne soumise à un vent moyen de $12 \mathrm{~m} / \mathrm{s}$. Cet enregistrement, qui illustre le caractère très fluctuant de la puissance générée par ce type d'éolienne, montre que cette puissance peut subir des variations de plus de $100 \mathrm{~kW}$ en 3 secondes et que la puissance nominale peut être dépassée de plus de $10 \%$. Ce type d'éolienne n'offre donc quasiment pas de possibilité de réglage de la puissance générée, d'autant plus que la connexion directe au réseau d'une génératrice asynchrone nécessite l'ajout de bancs de condensateurs afin de limiter la puissance réactive appelée à ce réseau.

Certaines éoliennes de ce type sont équipées d'un système à décrochage aérodynamique des pales (stall control). L'augmentation de la vitesse du vent s'accompagne automatiquement d'une diminution de l'angle de calage afin de permettre le décrochage aérodynamique de la turbine aux vitesses du vent plus grandes que la vitesse nominale. La structure du rotor de la turbine est ainsi plus simple, mais les possibilités de réglage de la puissance sont encore plus limitées. Une solution intermédiaire appelée «active stall » à également été développée. Elle garde les avantages du système «stall» (décrochage aérodynamique des pales) tout en intégrant un système d'orientation des pales simplifié [3], [10]. Les possibilités de réglages de la puissance générée par ce type d'éolienne restent toutefois marginales.

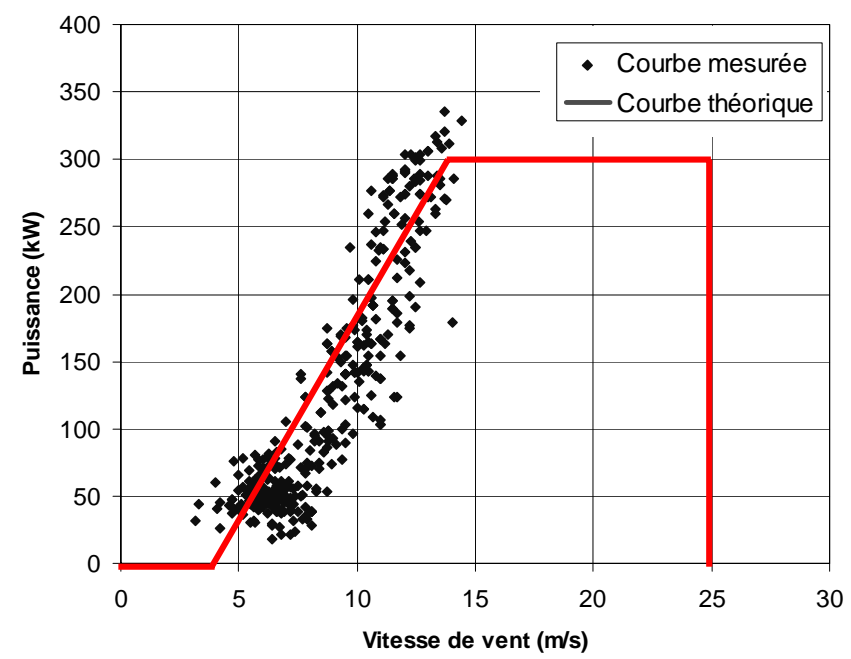

Fig. 3. Caractéristique de réglage d'une éolienne à vitesse fixe de $300 \mathrm{~kW}$.

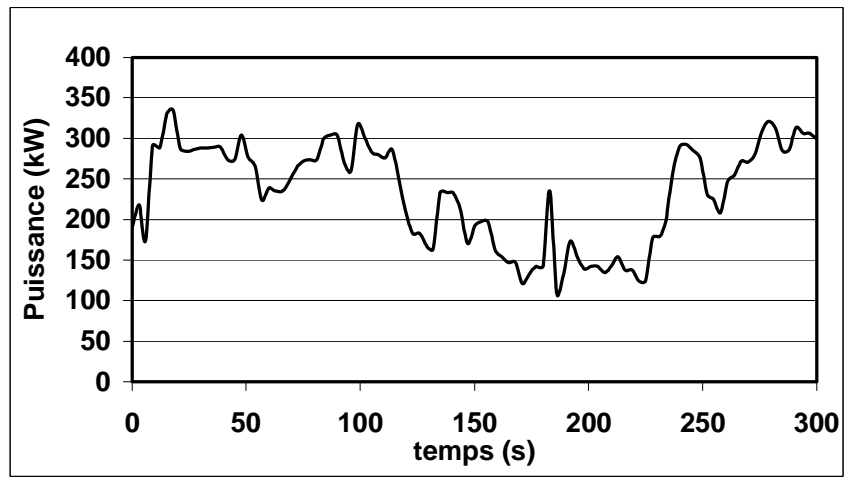

Fig. 4. Exemple de puissance générée par une éolienne à vitesse fixe de $300 \mathrm{~kW}$. 


\section{B. Eolienne à vitesse variable basée sur une machine asynchrone à double alimentation}

Sur la caractéristique en puissance d'une turbine (Fig. 5), le lieu du point représentant le maximum de la puissance convertie (représenté par la courbe en pointillés) peut être obtenu et parcouru en adaptant la vitesse de la turbine (courbe épaisse). Ainsi, afin de maximiser la puissance convertie, la vitesse de la turbine doit donc être adaptée par rapport à la vitesse du vent. C'est pourquoi les éoliennes de forte puissance raccordées aux réseaux moyenne et haute tension fonctionnent de plus en plus fréquemment à vitesse variable. Les principaux avantages des éoliennes à vitesse variable comparés aux générateurs à vitesse fixe sont les suivants :

- Elles augmentent la plage de fonctionnement, notamment pour les faibles vitesses de vent où le maximum de puissance est converti. Indirectement la disponibilité et la puissance générée du système sont augmentées.

- Elles nécessitent un système d'orientation des pales simplifié. En effet, la possibilité de contrôler la vitesse du générateur via le couple éléctromagnètique permet de réduire le rôle du système d'orientation des pales, qui interviendra essentiellement pour limiter la vitesse de la turbine et la puissance générée en présence de vitesses de vent élevées. En conséquence, pour de faibles vitesses de vent, l'angle d'orientation des pales devient fixe.

- Elles réduisent les efforts mécaniques de par le fait que lors de variations du vent, la vitesse de la turbine est adaptée. L' "élasticité" ainsi créée permet d'amoindrir l'incidence des rafales de vent sur la puissance générée pour ce domaine de fonctionnement.

- Elles réduisent le bruit lors des fonctionnements à faible puissance car la vitesse est alors lente.

- Elles permettent une meilleure intégration de l'éolienne dans le réseau électrique.

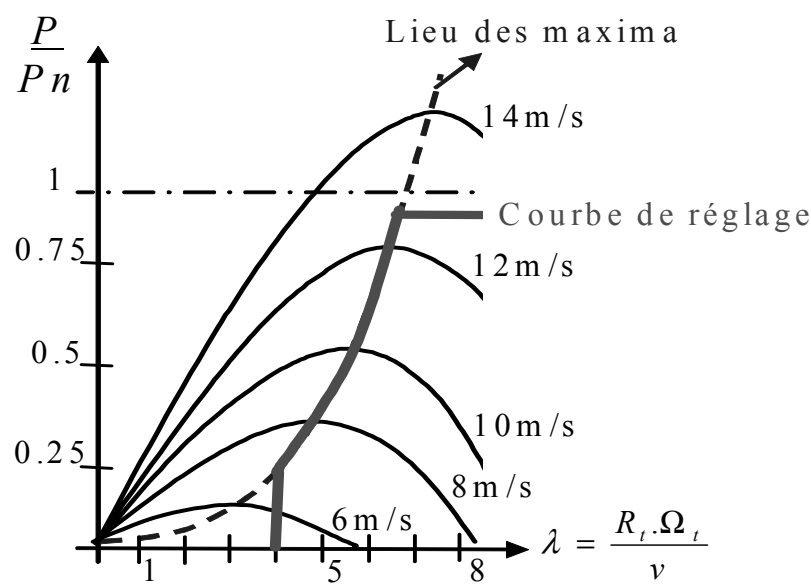

Fig. 5. Exemple de caractéristique de réglage de la vitesse.

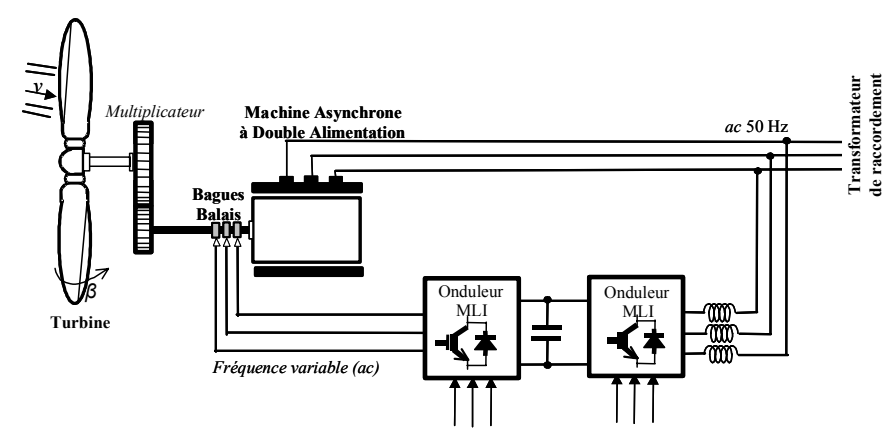

Fig. 6. Eolienne à vitesse variable basée sur une machine asynchrone à double alimentation.

Pour les générateurs à base de machine asynchrone, la différence entre la vitesse mécanique, $\Omega$, et la vitesse dite synchrone (imposée par la fréquence du réseau), $\Omega_{s}$, est définie par le glissement :

$$
g=\frac{\Omega_{s}-\Omega}{\Omega_{s}}
$$

Le glissement est fonction de la résistance que présente le circuit rotorique. Ainsi, pour les machines à circuit rotorique bobiné, une façon de rendre variable la vitesse mécanique de ce générateur est de rendre variable le glissement en modifiant la résistance du circuit rotorique. Plutôt que de dissiper cette puissance, il est beaucoup plus intéressant de la renvoyer sur le réseau au moyen de deux convertisseurs électroniques de puissance reliés par un bus continu (Fig. 6). Le circuit rotorique est rendu accessible grâce à un système de balaisbagues. En conséquence, la puissance transitant à travers le circuit rotorique est rendue variable et deux fonctionnements peuvent être distingués. Si la vitesse mécanique est supérieure à la vitesse synchrone $\left(g<0, \Omega>\Omega_{s}\right)$, un fonctionnement hypersynchrone est obtenu pour lequel la puissance est extraite $\mathrm{du}$ circuit rotorique et est envoyée sur le réseau à travers les convertisseurs de puissance. Sinon $\left(g>0, \Omega<\Omega_{s}\right)$, un fonctionnement hyposynchrone est obtenu et la puissance circule alors du réseau vers le circuit rotorique. Seule une variation de la vitesse de $+/-30 \%$ (correspondant à la valeur du glissement $g$ ) autour de la vitesse de synchronisme est acceptable; ceci va engendrer une limitation de la puissance circulant dans le circuit rotorique $(|g . P|)$. Comme seule une fraction de la puissance est rendue variable, la chaîne de conversion électronique est dimensionnée pour une moindre puissance et présente donc un coût réduit.

Le facteur de puissance peut être réglé car la génératrice peut être contrôlée pour fonctionner de façon similaire à celui d'un alternateur synchrone. En effet, la puissance active et la puissance réactive peuvent être contrôlées de façon indépendante grâce au convertisseur connecté sur le circuit électrique du rotor [14]. 
La Fig. 7 montre les zones de fonctionnement caractéristiques mesurées sur une éolienne de 1,5 MW [13] :

- La zone 2 est la zone pour laquelle une extraction maximale de la puissance est réalisée en adaptant la vitesse de la génératrice. La vitesse mécanique est très variable et correspond à une grande plage de variation de la puissance électrique produite. L'angle de calage des pales est maintenu constant, et seul le contrôle du couple électromagnétique de la génératrice est mis en œuvre dans cette zone.

- La zone 3 correspond à une vitesse mécanique quasiment constante de la génératrice. Dans cette zone la puissance générée est proportionnelle au couple (d'origine éolien) appliqué. La vitesse moyenne de la turbine est réglée par action sur l'orientation des pales, la puissance débitée au réseau est «lissée » impliquant un stockage inertiel dans les masses en rotation.

- Pour la zone 4, la puissance est limitée à sa valeur maximale $(1550 \mathrm{~kW})$ grâce au système d'orientation des pales.

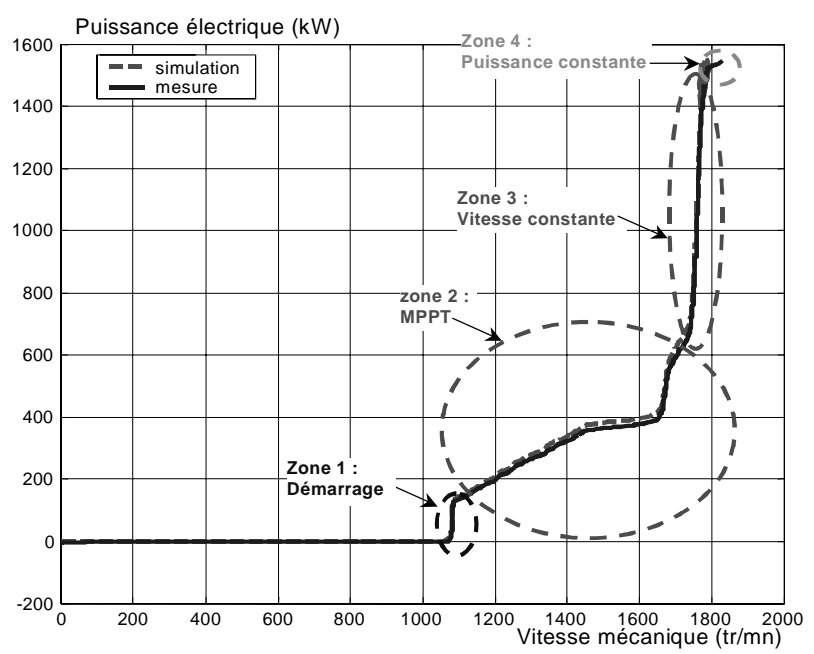

Fig. 7. Zones de fonctionnement caractéristiques d'une éolienne à vitesse variable basée sur une machine asynchrone.

Le contrôle de la puissance générée peut donc être réalisé en agissant sur l'orientation des pales, mais aussi en contrôlant le couple de la génératrice asynchrone au moyen du convertisseur de puissance connecté au rotor de celui-ci. Le contrôle de la puissance générée est dès lors nettement plus précis comme illustré sur la caractéristique puissance-vitesse du vent mesurée à la Fig. 8, à comparer aux mesures réalisées sur une éolienne à vitesse fixe (Fig. 3). La Fig. 9 représente la puissance générée par l'éolienne sur un intervalle de 10h alors qu'elle est soumise à un vent variant entre 2 et $16 \mathrm{~m} / \mathrm{s}$. On peut remarquer que la puissance maximale n'est pas dépassée.

Il existe également une technologie d'éolienne basée sur une génératrice asynchrone à rotor bobiné dans laquelle les bobinages rotoriques sont reliés à une résistance de dissipation via un redresseur à thyristor. Cette structure simplifiée permet un réglage limité de la vitesse, offre peu de possibilité de réglage de la puissance générée et présente un rendement moindre sur la plage de fonctionnement [3], [10].

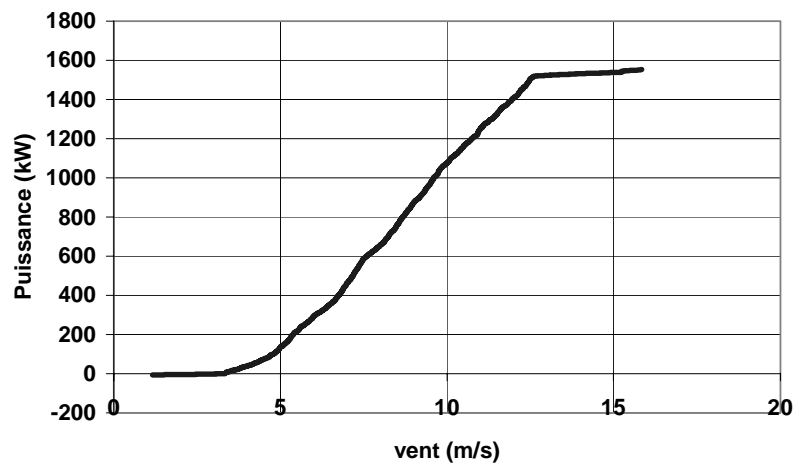

Fig. 8. Puissance totale générée mesurée sur une éolienne à vitesse variable de $1,5 \mathrm{MW}$ en fonction de la vitesse du vent.

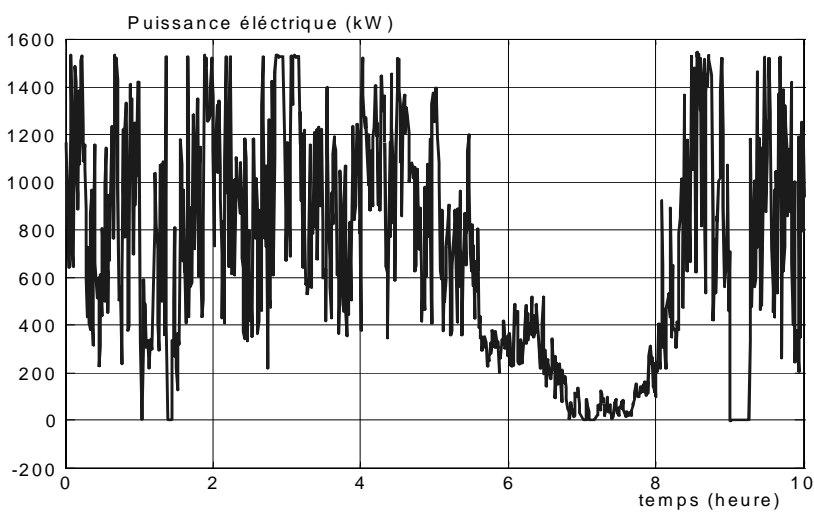

Fig. 9. Enregistrement de la puissance électrique générée par une éolienne à vitesse variable de 1,5 MW.

\section{Eolienne à vitesse variable basée sur une machine synchrone}

Les éoliennes basées sur une génératrice asynchrone à rotor bobiné présentent l'inconvénient de nécessiter un système de bagues et de balais et un multiplicateur, induisant des coûts significatifs de maintenance en particulier pour les projets offshore situés en milieu salin. Pour limiter ces inconvénients, certains constructeurs ont développé des éoliennes basées sur des machines synchrones à grand nombre de paire de pôles et couplées directement à la turbine, évitant ainsi le multiplicateur. Si de plus la génératrice est équipée d'aimants permanents, le système de bagues et de balais est éliminé. L'inconvénient de cette structure, représentée à la Fig. 10, est qu'elle nécessite pour sa connexion au réseau de convertisseurs de puissance dimensionnés pour la puissance nominale de la génératrice. Cet inconvénient est cependant un avantage du point de vue du contrôle de l'éolienne. En effet, l'interfaçage avec le réseau peut être entièrement contrôlé via le convertisseur connecté à ce réseau, tandis que le convertisseur connecté à la génératrice permet de contrôler la puissance générée par celle-ci en limitant le pitch control à une fonction de sécurité par grand vent. La courbe de réglage de ce type d'éolienne est généralement proche de celle présentée à la Fig. 5. 


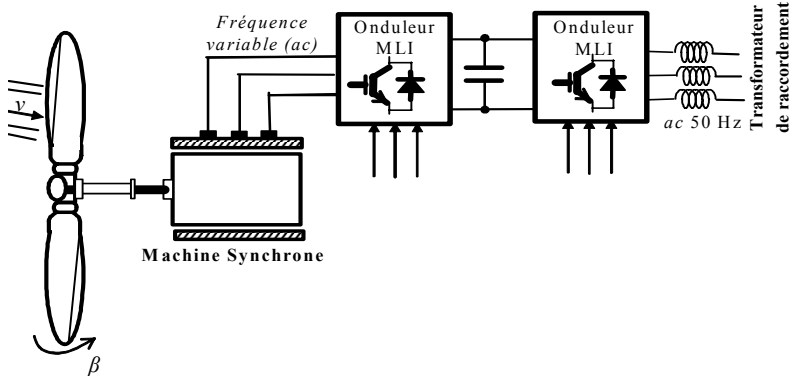

Fig. 10. Eolienne à vitesse variable basée sur une machine synchrone à grand nombre de paires de pôles.

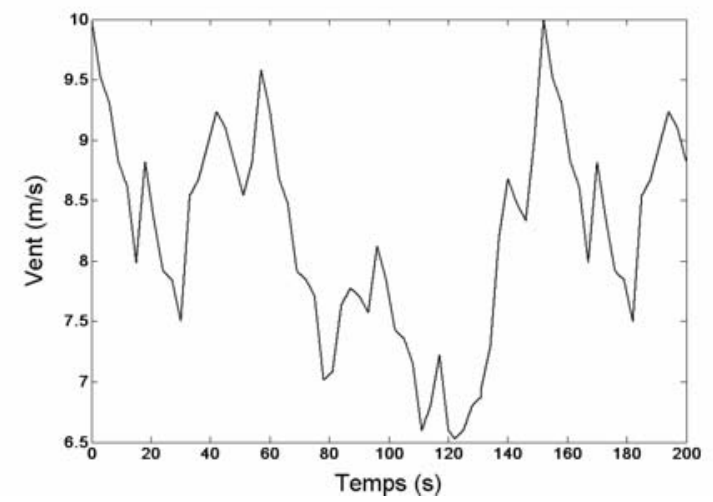

(a)

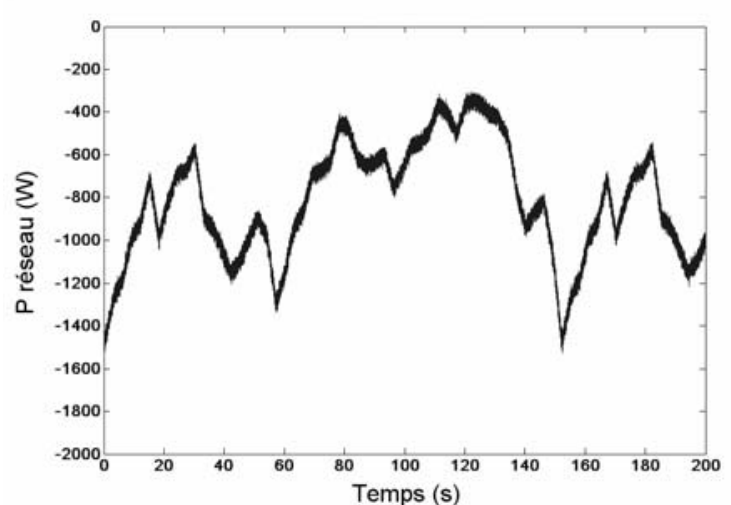

(b)

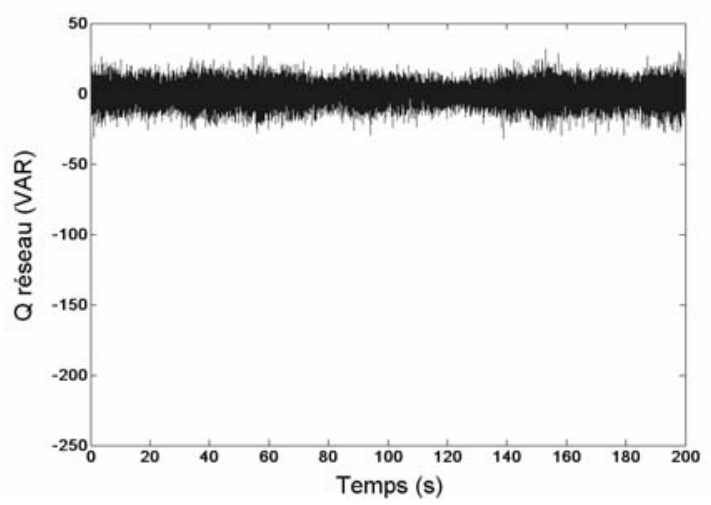

(c)

Fig. 11. Eolienne à vitesse variable émulée sur un banc d'essai de $3 \mathrm{~kW}$ (a) : Vitesse du vent. (b) : Puissance active en fonctionnement MPPT.

(c) : Puissance réactive maintenue nulle.
Les Figs. 11, 12 et 13 présentent des résultats expérimentaux mesurés sur une éolienne de ce type émulée sur un banc d'essais de $3 \mathrm{~kW}$ [15], [16]. Les Figs. 11a, 11b et 11c montrent la vitesse de vent très fluctuante à laquelle la turbine éolienne émulée est soumise, la puissance active renvoyée au réseau en extrayant le maximum d'énergie du vent, ainsi que la puissance réactive imposée nulle au point de connexion de l'éolienne (une puissance négative représente une puissance générée).

La puissance très fluctuante peut être lissée en contrôlant le couple de la génératrice afin d'imposer la vitesse de rotation de la turbine pour suivre une consigne de puissance. Cette technique revient à dégrader le coefficient de rendement $C p$ et est évidemment fonction du vent disponible.

La Fig. 12a montre la puissance active envoyée au réseau lorsque sa valeur de référence est imposée à $1 \mathrm{~kW}$ pour le même profil de vitesse de vent (Fig. 11a). Cette référence est relativement bien suivie tant que la vitesse du vent est suffisamment élevée. La Fig. 12b montre la puissance réactive qui est toujours maintenue nulle.

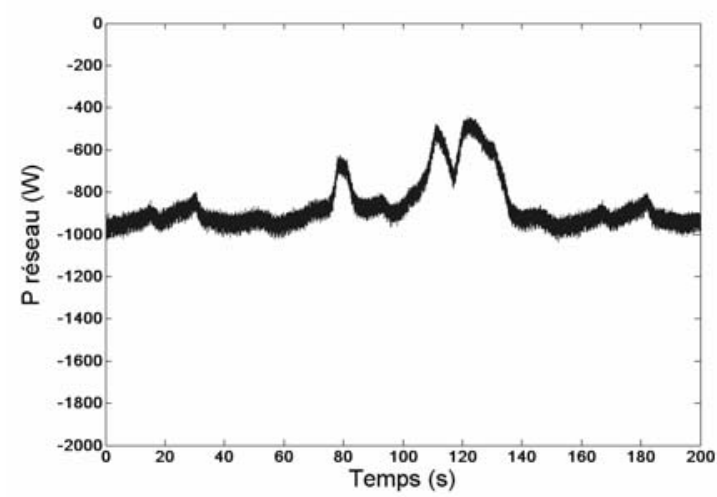

(a)

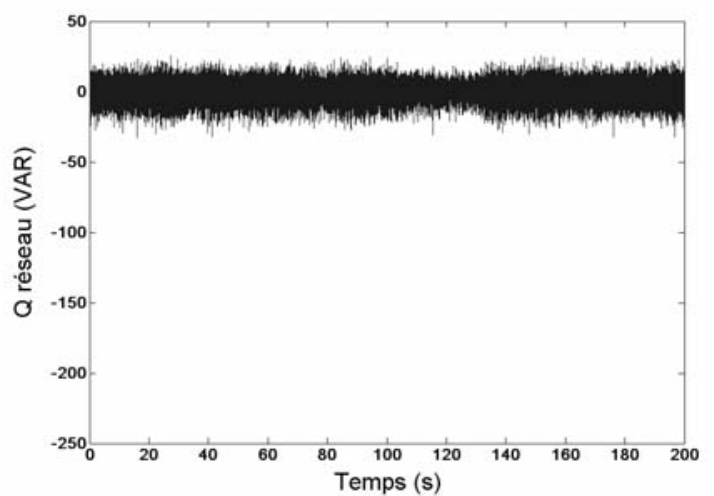

(b)

Fig. 12. Eolienne à vitesse variable émulée sur un banc d'essai de $3 \mathrm{~kW}$. (a) : Puissance active lissée. (b) : Puissance réactive maintenue nulle.

L'essai illustré à la Fig. 13a est similaire à celui de la Fig. $12 \mathrm{a}$ en ce qui concerne la puissance active ; par contre un échelon de 200 VAR est imposé à la référence de la puissance réactive (Fig. 13b). Le réglage des puissances active et réactive apparaît bien découplé. Il faut toutefois noter que la 
puissance réactive pouvant effectivement être générée ou absorbée par l'éolienne est limitée par la valeur de la puissance active générée et par le niveau de tension du bus continu [17].

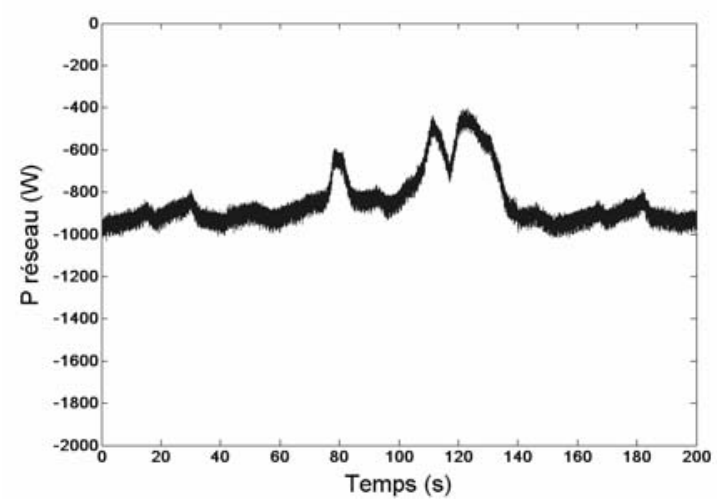

(a)

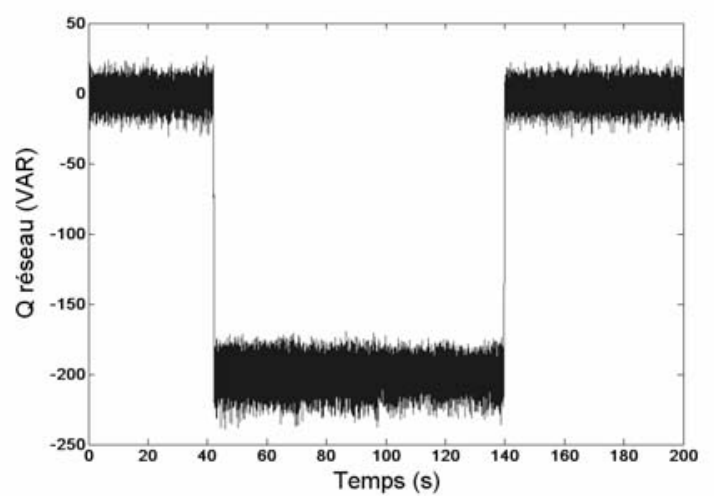

(b)

Fig. 13. Eolienne à vitesse variable émulée sur un banc d'essai de $3 \mathrm{~kW}$. (a) : Puissance lissée. (b) : Puissance réactive avec échelon de 200 VAR.

Ces essais illustrent les possibilités de réglages des puissances active et réactive générées par les éoliennes à vitesse variable, laissant entrevoir un potentiel de participation au réglage de la tension et de la fréquence du réseau.

\section{FERME D'EOLIENNES}

Les éoliennes sont regroupées en ferme. Les éoliennes à vitesse fixe et celles basées sur une machine asynchrone à double alimentation sont reliées au réseau au moyen d'une liaison à courant alternatif. Les éoliennes basées sur une machine synchrone peuvent être reliées de la même façon (Fig. $14)$; la présence d'un bus continu dimensionné pour la tension nominale de la génératrice permet également d'envisager une connexion des éoliennes entre - elles via une liaison à courant continu (Fig. 15). Cette architecture, imaginée pour les fermes éoliennes off-shore [18] interfacées avec le réseau via un seul convertisseur continu - alternatif, permet d'envisager, par exemple, une meilleure participation de la ferme éolienne au réglage de la tension au point de connexion. D'autres variantes d'architecture de ferme éolienne sont présentées dans [10].

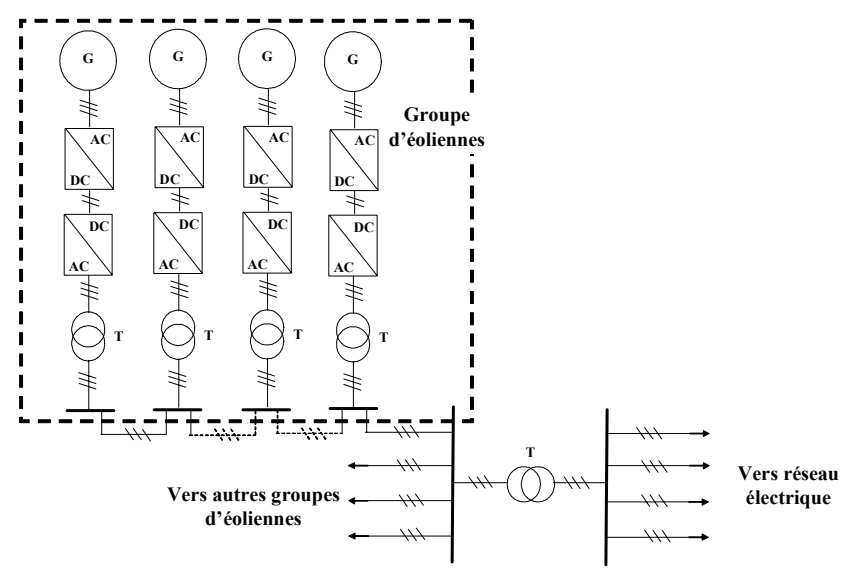

Fig. 14. Exemple d'architecture d'une ferme d'éoliennes à vitesse variable avec des liaisons en tensions alternatives.

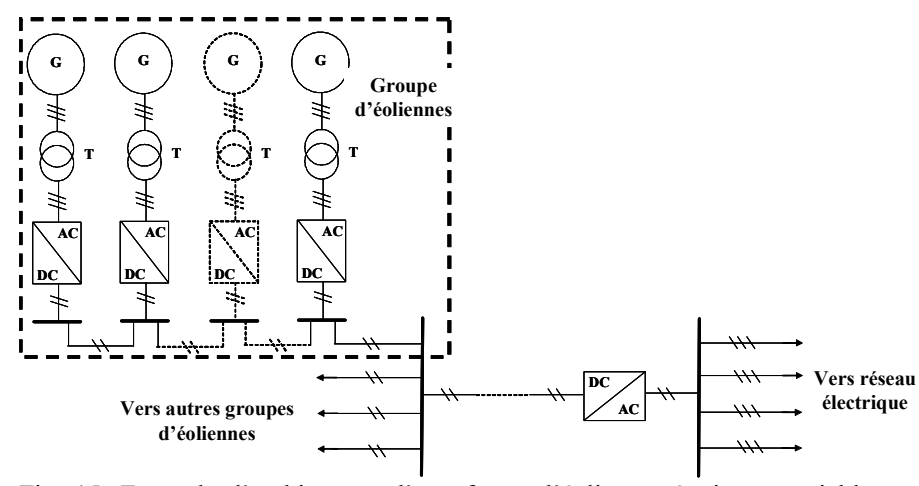

Fig. 15. Exemple d'architecture d'une ferme d'éoliennes à vitesse variable avec des liaisons en tensions continues.

$\mathrm{Du}$ fait que les éoliennes dans une ferme sont positionnées sur un certain espace (par exemple la distance entre deux éoliennes de $300 \mathrm{~kW}$, placées l'une derrière l'autre, doit être au moins de $168 \mathrm{~m}$ ), ces éoliennes sont soumises à des vents ayant des vitesses sensiblement différentes. Ce foisonnement induit que la puissance générée sur le réseau par la ferme apparaît plus lissée que celle générée par une seule éolienne. A partir de l'enregistrement réalisé pour une éolienne de $300 \mathrm{~kW}$ (présenté à la Fig. 4), les puissances générées par des groupes de 3 et 10 éoliennes ont été reconstituées et sont présentées respectivement aux Figs. 16 et 17. La comparaison des Figs. 4, 16 et 17 montre un lissage significatif de la puissance générée (au moins en valeur relative) lorsque le nombre d'éolienne de la ferme augmente.

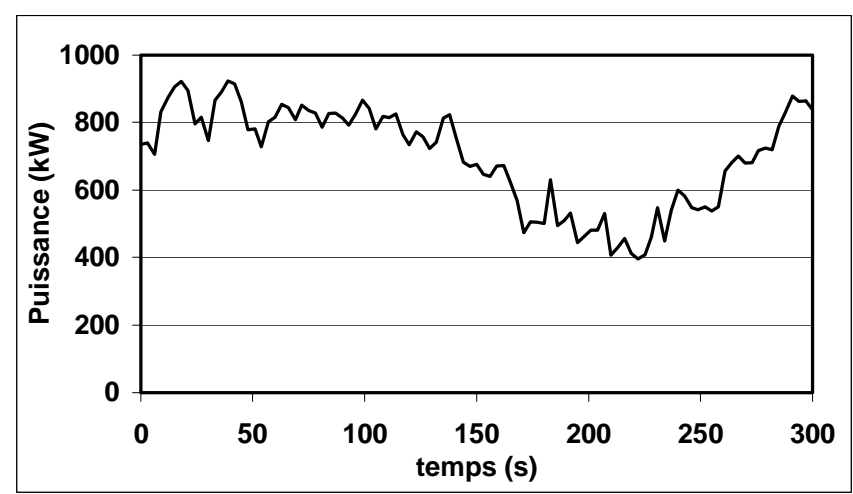

Fig. 16. Puissance totale générée par une ferme de trois éoliennes de 300 kW. 


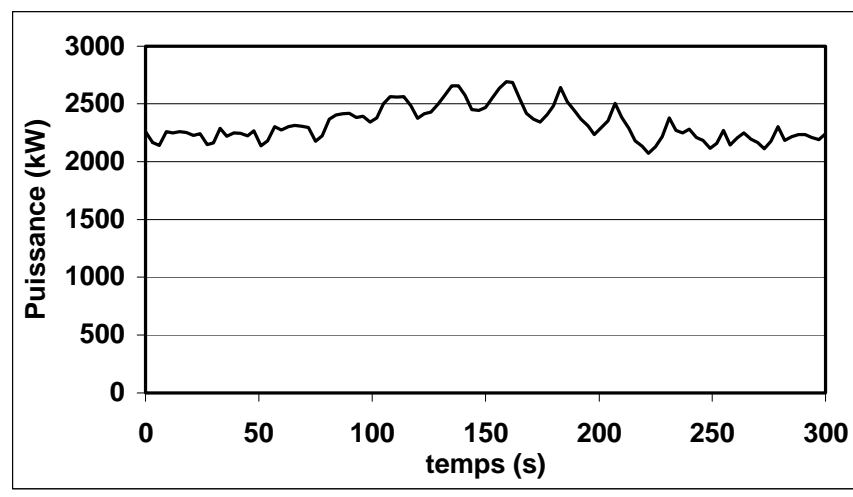

Fig. 17. Puissance totale générée par une ferme de dix éoliennes de $300 \mathrm{~kW}$.

\section{TENSION DE RACCORDEMENT}

Les modalités de raccordement au réseau électrique des installations de production, et notamment les contraintes techniques, sont définies par des textes réglementaires tels que décrets et arrêtés. Les contraintes techniques dépendent principalement de la puissance à raccorder qui définit le réseau de connexion. Le tableau 1 donne les niveaux de tension de raccordement des installations en fonction de leur puissance, en France.

\begin{tabular}{|r|c|c|c|}
\hline $\begin{array}{r}\text { Type } \\
\text { de réseau }\end{array}$ & Plage de tension & $\begin{array}{c}\text { Niveaux de } \\
\text { tension }\end{array}$ & Puissance \\
\hline BT & BT monophasé & $230 \mathrm{~V}$ & $\mathrm{~S} \leq 18 \mathrm{kVA}$ \\
\hline BT & $\mathrm{BT}$ triphasé & $400 \mathrm{~V}$ & $\mathrm{~S} \leq 250 \mathrm{kVA}$ \\
\hline $\begin{array}{r}\text { MT } \\
\text { (HTA) }\end{array}$ & $1 \mathrm{kV}<\mathrm{U} \leq 50 \mathrm{kV}$ & $15 \mathrm{kV}, 20 \mathrm{kV}$ & $\mathrm{P} \leq 12 \mathrm{MW}$ \\
\hline $\begin{array}{c}\text { HT } \\
\text { (HTB1) }\end{array}$ & $50 \mathrm{kV}<\mathrm{U} \leq 130 \mathrm{kV}$ & $63 \mathrm{kV}, 90 \mathrm{kV}$ & $\mathrm{P} \leq 50 \mathrm{MW}$ \\
\hline $\begin{array}{r}\text { HT } \\
\text { (HTB2) }\end{array}$ & $130 \mathrm{kV}<\mathrm{U} \leq 350 \mathrm{kV}$ & $150 \mathrm{kV}, 225 \mathrm{kV}$ & $\mathrm{P} \leq 250 \mathrm{MW}$ \\
\hline $\begin{array}{r}\text { HT } \\
\text { (HTB3) }\end{array}$ & $350 \mathrm{kV}<\mathrm{U} \leq 500 \mathrm{kV}$ & $400 \mathrm{kV}$ & $\mathrm{P}>250 \mathrm{MW}$ \\
\hline
\end{tabular}

Tableau 1 - Niveaux de tension de raccordement des installations en fonction de leur puissance.

Les installations de production relèvent donc:

- du réseau public de transport si la puissance installée est supérieure à $12 \mathrm{MW}$, le raccordement s'effectue alors à un niveau de tension supérieur ou égal à $63 \mathrm{kV}$ (domaine de la HTB) ;

- du réseau public de distribution si la puissance installée est inférieure ou égale à $12 \mathrm{MW}$, le raccordement s'effectue alors à un niveau de tension inférieur ou égal à $20 \mathrm{kV}$ (domaine de la HTA et de la BT).

Les modalités de raccordement au réseau HTA français sont commentées dans [19], [20]. Dans la suite de cet article, nous synthétisons et commentons les modalités de raccordement au réseau HTB français.

\section{CONTRAINTES DE RACCORDEMENT DE L'EOLIEN AU RESEAU DE TRANSPORT}

Les prescriptions techniques relatives au raccordement au réseau de transport d'une installation de production d'énergie électrique sont précisées dans le décret [8] et l'arrêté [9]. Le décret [8] présente quelques considérations générales sur ces contraintes de raccordement :

- En fonction de leur type, de leur puissance et de leur tension de raccordement, les installations de production doivent être conçues pour pouvoir contribuer, pour des durées limitées, au soutien du système électrique lorsqu'il est en régime exceptionnel ou en situation de défaut d'isolement.

- En fonction de leur type, de leur puissance et de leur tension de raccordement, les installations de production doivent avoir des capacités constructives de fourniture de services auxiliaires nécessaires pour que le fonctionnement du système électrique soit sûr. Ces services comprennent :

. les réglages primaire et secondaire de la tension;

- les réglages primaire et secondaire de la fréquence ;

. le fonctionnement en réseau séparé ;

. le renvoi de tension et la participation à la reconstitution du réseau.

- les installations de production doivent être équipées d'un dispositif qui permet de les coupler au réseau public de transport. Les installations doivent en outre être conçues pour que la stabilité de leur fonctionnement soit assurée compte tenu des caractéristiques de leur raccordement au réseau et doivent être équipées des régulations de tension et de fréquence (c'est-à-dire de la vitesse pour les groupes tournants) qui sont nécessaires pour atteindre cet objectif. Des pertes de stabilité ne doivent pas être à l'origine d'une dégradation de la qualité de l'électricité sur le réseau public de transport ou de la perturbation des conditions de son exploitation.

- Les installations de production doivent être capables de recevoir et d'exécuter, dans les délais appropriés précisés dans la convention d'exploitation, les ordres de conduite et de sauvegarde en provenance du gestionnaire du réseau de transport.

L'arrêté [9] précise ces contraintes de raccordement en fonction du type d'installation. Sans prétendre être exhaustifs, nous allons évoquer quelques unes de ces contraintes dans la suite de cette section et dans la section suivante.

\section{A. Réglage de la tension}

Toutes les unités de production, y compris les éoliennes, doivent pouvoir fonctionner dans un domaine de fonctionnement déterminé par un graphique avec en ordonnée la tension $(U)$ et en abscisse le rapport entre la puissance réactive et la puissance active maximale $\left(Q / P_{\max }\right)$. Un exemple de domaine de fonctionnement dénommé trapèze $[U, Q]$ est représenté à la Fig. 18. 


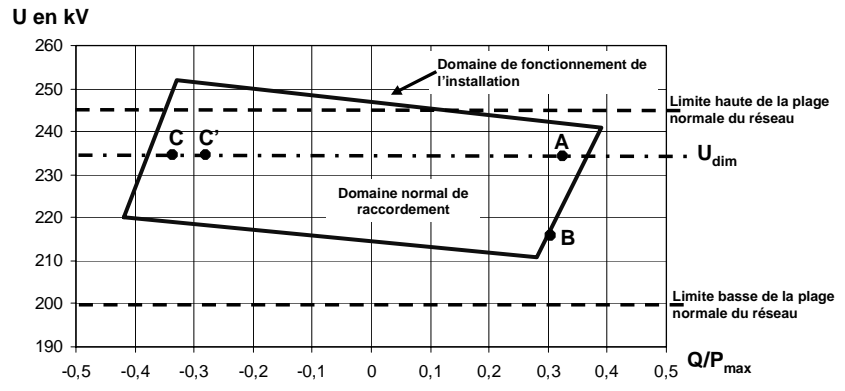

Fig. 18. Exemple de domaine normal de fonctionnement d'une installation de production.

Les unités de production doivent assurer un contrôle de la tension et/ou de la puissance réactive au point de livraison. Trois types de réglage primaire sont possibles:

-Type 1 : réglage à puissance réactive constante ;

-Type 2 : réglage de la tension à une valeur variant linéairement en fonction de la puissance réactive avec une pente ajustable ;

-Type 3 : réglage de la tension selon une consigne asservie aux ordres provenant du réglage secondaire de la tension.

Les installations raccordées aux réseaux HTB2 et HTB3 doivent pouvoir participer au réglage secondaire de la tension de leur zone, si le gestionnaire du réseau le demande. Ces installations doivent disposer d'un réglage primaire de la tension de type 2 ou 3.

\section{B. Réglage de la fréquence}

La participation au réglage de la fréquence n'est pas exigée pour des installations de production mettant en œuvre de l'énergie fatale telles que les fermes éoliennes. Il est néanmoins intéressant de noter les contraintes imposées aux autres installations :

- Les installations d'une puissance supérieure ou égale à 40MW doivent participer au réglage primaire de fréquence ;

- Les installations d'une puissance supérieure ou égale à 120MW doivent participer au réglage secondaire de fréquence.

Le fait de ne pas participer au réglage de fréquence limitera à terme le taux de pénétration de l'éolien, car ce réglage est reporté sur les groupes traditionnels. En cas de forte pénétration de l'éolien, les exigences en terme de participation au réglage de fréquence de l'éolien pourraient donc évoluer. Le problème étant évidemment l'existence d'une réserve primaire associé à l'éolien.

Il en est de même pour le fonctionnement en réseau séparé et pour la participation à la reconstitution du réseau qui ne sont actuellement pas exigées pour les fermes éoliennes.

\section{Couplage et perturbations}

Le couplage de l'installation au réseau doit respecter des contraintes sur la fréquence, l'amplitude et la phase de la tension et ne doit pas affecter la stabilité du réseau.

Les perturbations produites par l'installation au point de raccordement doivent être limitées en ce qui concerne les àcoups de tension, le flicker, les déséquilibres et les harmoniques.

Un système de communication entre le producteur et le gestionnaire du réseau est à prévoir.

\section{PROBLEMES INDUITS PAR L'INTEGRATION DE L'EOLIEN DANS LE RESEAU DE TRANSPORT}

Afin d'assurer la sécurité du réseau, le gestionnaire de ce réseau doit veiller à éviter la surcharge thermique des lignes et la rupture de synchronisme, assurer la stabilité de la fréquence (c'est-à-dire assurer l'équilibre production - consommation) et la stabilité de la tension [21].

\section{A. Prévision de la production}

Le problème majeur de l'éolien est l'incertitude sur les prévisions de la vitesse du vent sur les sites éoliens induisant une incertitude sur la production des fermes éoliennes. Dans [3], des exemples de mauvaises prévisions concernant le Danemark sont illustrées. Les erreurs de prévisions à $24 \mathrm{~h}$ peuvent atteindre des valeurs de plus de $50 \%$ en négatif (sousproduction) ou en positif (sur-production) avec des changements de signe rapides en cours de journée. Des capacités de production de réserves contrôlables sont alors nécessaires pour pallier ces incertitudes, en particulier pour satisfaire la demande en période de pointe. L'opérateur ELTRA, gérant le réseau à l'ouest du Danemark intégrant une forte capacité éolienne (2400 MW en 2003), a connu des situations critiques dues à une sur-production éolienne dont l'évacuation vers le réseau nordique (Nordel) ou vers le réseau UCTE (via l'Allemagne) posait problème du fait que les lignes d'interconnexion étaient déjà aux limites de leur capacité.

La prévision est globalement bonne en ce qui concerne l'amplitude mais présente une incertitude sur le moment où ce niveau de production sera atteint [22].

\section{B. Capacité d'accueil du réseau}

La capacité des lignes et des postes est limitée. Cette limitation peut constituer dans le cas de l'éolien un problème aigu, car les lieux de production (sites ventés) sont souvent éloignés des lieux de consommation. L'adaptation et le renforcement des postes peuvent concerner les postes HTAHTB suite au développement de l'éolien dans le réseau de distribution (modification des protections, augmentation de la puissance de court-circuit,...). Afin d'éviter la congestion des lignes de transport et d'assurer la sécurité du réseau, de nouvelles lignes devraient être construites en particulier aux interconnexions entre les réseaux gérés par des opérateurs différents. Une étude prospective réalisée dans la zone gérée 
par l'opérateur allemand EON Netz prévoit le développement de $1000 \mathrm{~km}$ de lignes supplémentaires d'ici 2016 pour une capacité éolienne de $16000 \mathrm{MW}$, dont une partie importante en off-shore, et ce pour un montant de 550 millions d'Euros [3]. Il faut cependant noter que le délai de renforcement d'un poste peut atteindre 5 ans et le délai de construction d'une nouvelle ligne peut atteindre 10 ans et faire l'objet d'oppositions importantes de la part des populations. RTE a évalué la capacité d'accueil du réseau français, sans adaptation importante, entre 6000 et $7000 \mathrm{MW}$ d'éolien [2].

\section{Déconnexions intempestives}

Les générateurs éoliens, tout comme la majorité des générateurs décentralisés, sont très sensibles aux perturbations du réseau et ont tendance à se déconnecter rapidement lors d'un creux de tension (dès que la valeur de la tension est inférieure à $80 \%$ de la tension nominale) ou lors d'une variation de la fréquence. Lors du black-out italien du 28 septembre 2003, 3400 MW de productions décentralisées se sont déconnectés lorsque la fréquence du réseau a atteint 49 $\mathrm{Hz}$ [23]. L'opérateur EON Netz prévoit que le risque de déconnexion de $3000 \mathrm{MW}$ d'éolien suite à un défaut important sur le réseau $380 \mathrm{kV}$ au nord de l'Allemagne est bien réel [3], pouvant ainsi induire un déséquilibre productionconsommation aggravant les conséquences d'un défaut qui aurait pu être éliminé normalement.

Afin d'éviter un déclenchement simultané de toute ou partie de la production éolienne sur un défaut normalement éliminé, il est demandé aux éoliennes installées depuis 2003 de pouvoir rester connectées au réseau en cas de baisse de tension et de variation de fréquence suivant des contraintes pouvant varier d'un opérateur à l'autre. Par exemple, RTE demande que les éoliennes restent connectées au réseau tant que le creux de tension reste supérieur au gabarit présenté à la Fig. 19 (valable pour les réseaux de répartition: réseaux de transport régionaux à $225 \mathrm{kV}, 90 \mathrm{kV}$ et $63 \mathrm{kV}$ ) [9], [24].

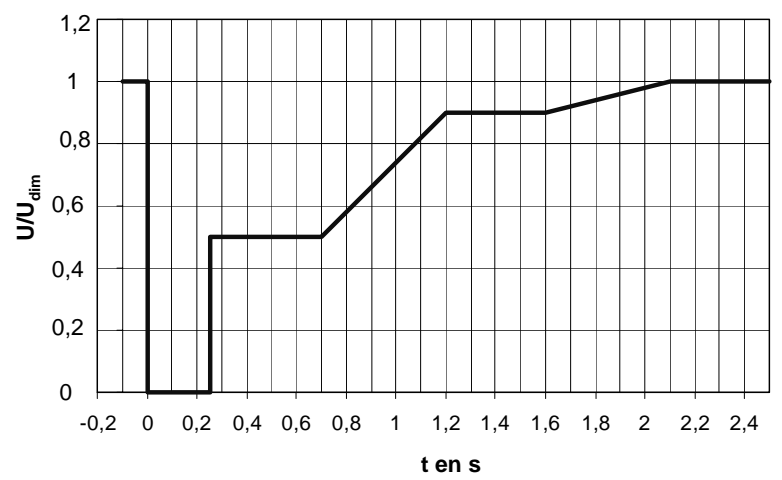

Fig. 19. Gabarit de tension auquel doivent satisfaire les éoliennes connectées au réseau de répartition.

Concernant les variations de fréquence, l'arrêté [9] précise que les installations de production doivent rester connectées pour des durées limitées dans les plages exceptionnelles de fréquence situées entre 47 et $52 \mathrm{~Hz}$. De plus, même si l'installation ne participe pas à la constitution des réserves comme c'est le cas de l'éolien, elle doit être capable de réduire la puissance produite lorsque la fréquence dépasse un seuil réglable entre 50,5 et $51 \mathrm{~Hz}$.

\section{Qualité de l'électricité}

\section{1) La tension}

Pour le cas très simple d'une charge alimentée à travers une ligne par une source de tension constante (Fig. 20), on peut écrire de façon approchée que la chute de tension dans la ligne $\left(\Delta V=V_{1^{-}} V_{2}\right)$ est égale à

$$
\Delta V=\frac{r P+x Q}{V_{2}}
$$

avec $r$ la résistance des conducteurs en $\Omega, x$ la réactance de la ligne en $\Omega, P$ et $Q$ respectivement les puissances active et réactive transitant dans la ligne.

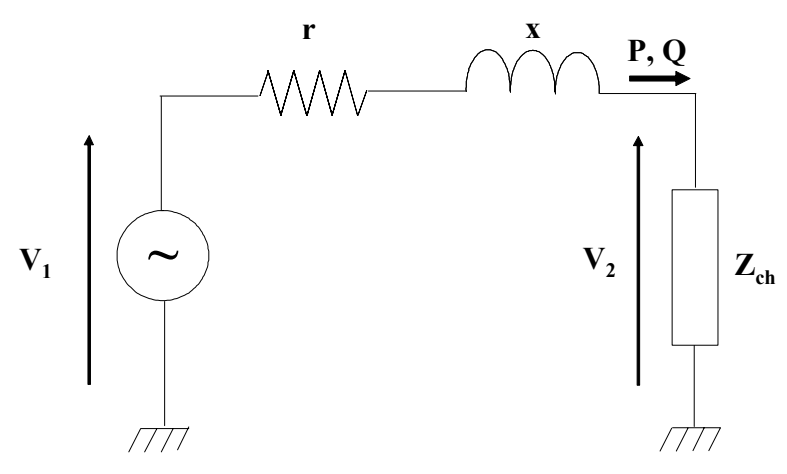

Fig. 20. Schéma équivalent simplifié d'une ligne.

Pour les lignes de Très Haute tension (THT), $x \geq 10 r$; l'expression (3) peut donc se simplifier :

$$
\Delta V=\frac{x Q}{V_{2}}
$$

La puissance active générée par les éoliennes est par nature fluctuante, ce qui tend d'après l'expression (3) à induire des fluctuations de tension dans la zone du réseau proche du point de connexion de ces éoliennes. Dans le réseau de transport constitué de ligne HT et THT (ou HTB), l'expression (4) montre que les variations de tension sont essentiellement induites par les transits de puissance réactive, contrairement aux réseaux de distribution. C'est pourquoi, il est demandé aux éoliennes actuellement connectées au réseau de transport de participer au réglage de la tension via un réglage de la puissance réactive. Ce réglage est possible avec les éoliennes connectées au réseau via de l'électronique de puissance [25] (Figs. 6-10), mais pas avec les éoliennes à vitesse fixe dont la génératrice asynchrone est directement couplée au réseau. 
Cette dernière consommant une puissance réactive significative, nécessite l'ajout de condensateurs de compensation.

Les variations régulières de tension sont appelées flicker. Dans le cas des éoliennes, ces variations sont dues aux fluctuations de la vitesse du vent, aux limites mécaniques de l'éolienne (par exemple du pitch control) et à l'effet d'ombre causé par le passage des pales devant le mât. A nouveau, ce sont les éoliennes à vitesse fixe qui sont les plus défavorables du point de vue de ces phénomènes. La technologie d'éolienne la mieux adaptée pour limiter l'impact sur le réseau de ces variations est celle complètement interfacée avec le réseau via de l'électronique de puissance permettant ainsi un certain découplage entre la turbine et le réseau. Les éoliennes, interfacées au réseau via des convertisseurs d'électronique de puissance, génèrent cependant des harmoniques. Les convertisseurs totalement commandés équipés de transistors IGBT, actuellement les plus utilisés, génèrent des harmoniques haute fréquence (plusieurs $\mathrm{kHz}$ ), mais ces harmoniques peuvent être assez aisément limités contrairement aux convertisseurs partiellement commandés, équipés de thyristors, générant des harmoniques de basse fréquence nécessitant des filtres conséquents pour les éliminer.

\section{2) La fréquence}

Le réglage primaire de la fréquence est basé sur le constat qu'un déséquilibre entre la production et la consommation induit une variation de fréquence due à la variation de vitesse des groupes alternateurs classiques. Le réglage primaire de fréquence est réalisé automatiquement au niveau des groupes de production. Il assure une correction rapide, en quelques secondes, et décentralisée des écarts offre-demande. Ce réglage suit une relation linéaire ente la fréquence (imposée par la vitesse des groupes tournants) et la puissance (Fig. 21).

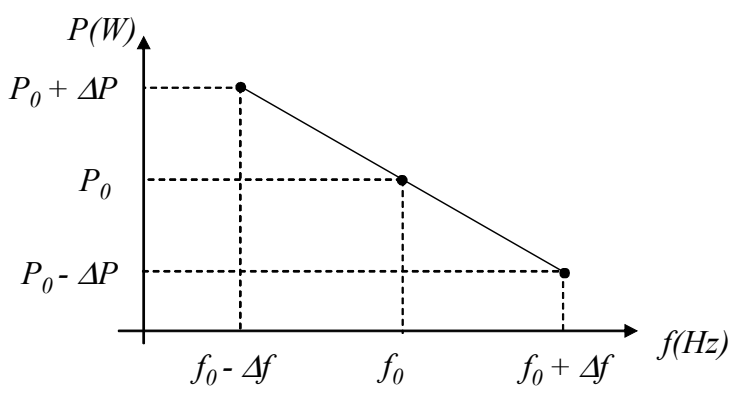

Fig. 21. Réglage primaire de fréquence.

$P_{0}$ est la puissance de consigne à la fréquence de référence $f_{0}$, et $\Delta P$ la variation de puissance induite par la variation de fréquence $\Delta f$.

L'adaptation rapide de la production à la consommation faite par le réglage primaire laisse, en fin d'action, un écart de fréquence. De plus, comme la fréquence est commune aux réseaux interconnectés (par exemple le réseau synchrone de l'UCTE), ce réglage provoque des variations de transit entre les pays. Ceux - ci sont l'expression de la «solidarité » entre réseaux pour contenir les aléas (perte de groupe de production). Le rôle du réglage secondaire est alors, en quelques minutes (15 minutes en UCTE), de ramener la fréquence à sa valeur nominale et de ramener les échanges entre partenaires à leurs valeurs contractuelles. C'est l'expression du principe de «responsabilité » : la correction de l'aléa étant du ressort du réseau qui en a été le siége.

Les variations rapides de la puissance générée par les éoliennes (pouvant atteindre quelques centaines de $\mathrm{kW}$ en quelques dizaines de seconde), tout comme les variations de charge, peuvent donc induire des fluctuations de la fréquence $\mathrm{du}$ réseau et activer le réglage primaire des groupes tournants. Cependant, tant que le taux de pénétration de l'éolien reste faible, cette influence peut être considérée comme négligeable. Dans le cas contraire, afin d'assurer la stabilité du réseau, une participation au réglage primaire des éoliennes sera à envisager avec des solutions qui restent à imaginer. Déjà actuellement, lorsque la production est supérieure à la consommation, donc lorsque la fréquence est supérieure à $50 \mathrm{~Hz}$, il peut être demandé aux éoliennes de réduire leur production [3].

\section{SimULATIONS}

\section{A. Impact d'une ferme d'éoliennes}

Le premier exemple traité, au moyen du logiciel Eurostag, considère un réseau maillé simple alimenté par de la production classique (Fig. 22). Celle-ci est divisée en deux ensembles, représentés par deux unités équivalentes: un premier groupe d'unités assure le réglage primaire (SR6MVA), un second ensemble d'unités fournit le réglage secondaire ( $\mathrm{SNR}$ - 22,5 MVA). Le système est dimensionné pour assurer la continuité de service en cas de perte du groupe ne participant pas au réglage de la fréquence (SF - 1,5 MVA), produisant $1 \mathrm{MW}$. La consommation est de l'ordre de $13 \mathrm{MW}$.

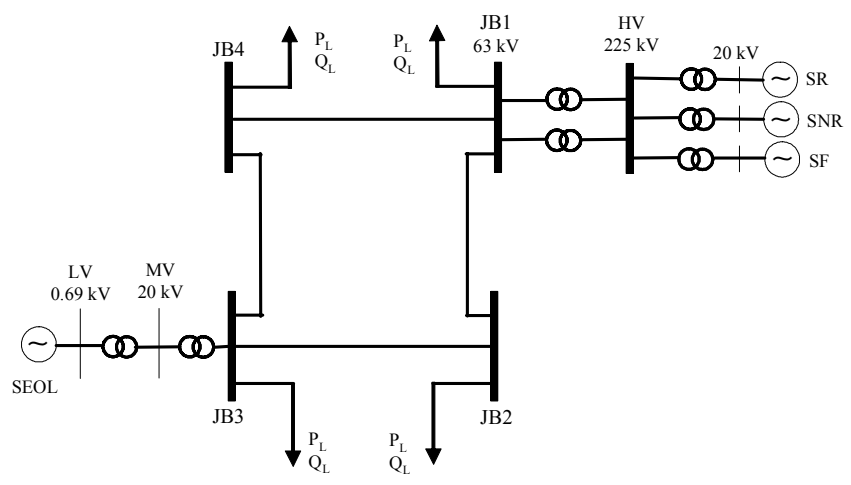

Fig. 22. Configuration du réseau maillé.

A la suite du déclenchement du groupe de production SF, la fréquence du système baisse jusque $49,4 \mathrm{~Hz}$. Elle se rétablit sous l'influence des réglages primaire et secondaire. La Fig. 23 montre l'évolution des points de fonctionnement des groupes de production et la courbe d'évolution de la fréquence. Le profil de fréquence montre que le système considéré (pouvant 
s'apparenter à un système isolé) présente un réglage de fréquence satisfaisant.

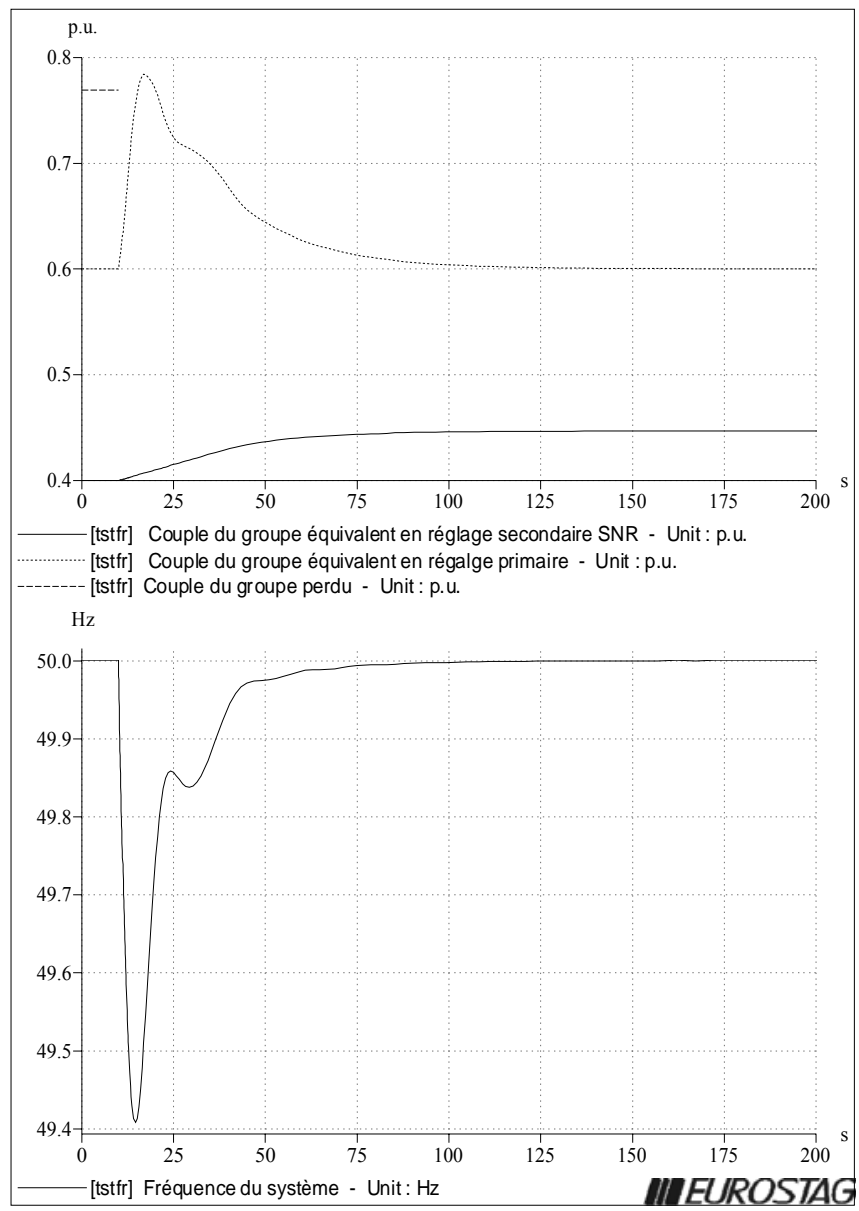

Fig. 23. Réponse du système à la perte du groupe SF.

Dans ce réseau sont connectées 5 éoliennes à vitesse fixe d'une puissance nominale de $750 \mathrm{~kW}$ chacune. Elles sont soumises à un profil de vent enregistré sur un site éolien à Dunkerque sur une base d'un point par 3 secondes. Ce profil de vent est «lissé » par un premier ordre de constante de temps 0.5 seconde et par un filtrage numérique de 5 retards de 0.3 à 2.7 secondes.

Deux situations distinctes ont été simulées. La première applique ce profil de vent aux 5 éoliennes. La seconde modélisation introduit un décalage de temps entre les différentes éoliennes (ceci ne doit être considéré que comme une façon de représenter la variabilité du vent appliqué à chaque éolienne). Le premier tracé de la Fig. 24 montre l'enregistrement du vent et le profil filtré et décalé d'environ $1 \mathrm{~m} . \mathrm{s}^{-1}$. Le deuxième tracé montre la variation de la production éolienne totale et l'évolution correspondante de la production $\mathrm{du}$ groupe réglant SR quand le même profil de vent est considéré pour les 5 éoliennes. Le dernier tracé montre la réduction significative des variations quand un facteur de non simultanéité est pris en compte (simulé ici par un décalage temporel du profil de vent imposé à chaque éolienne de l'ordre de 60 secondes).

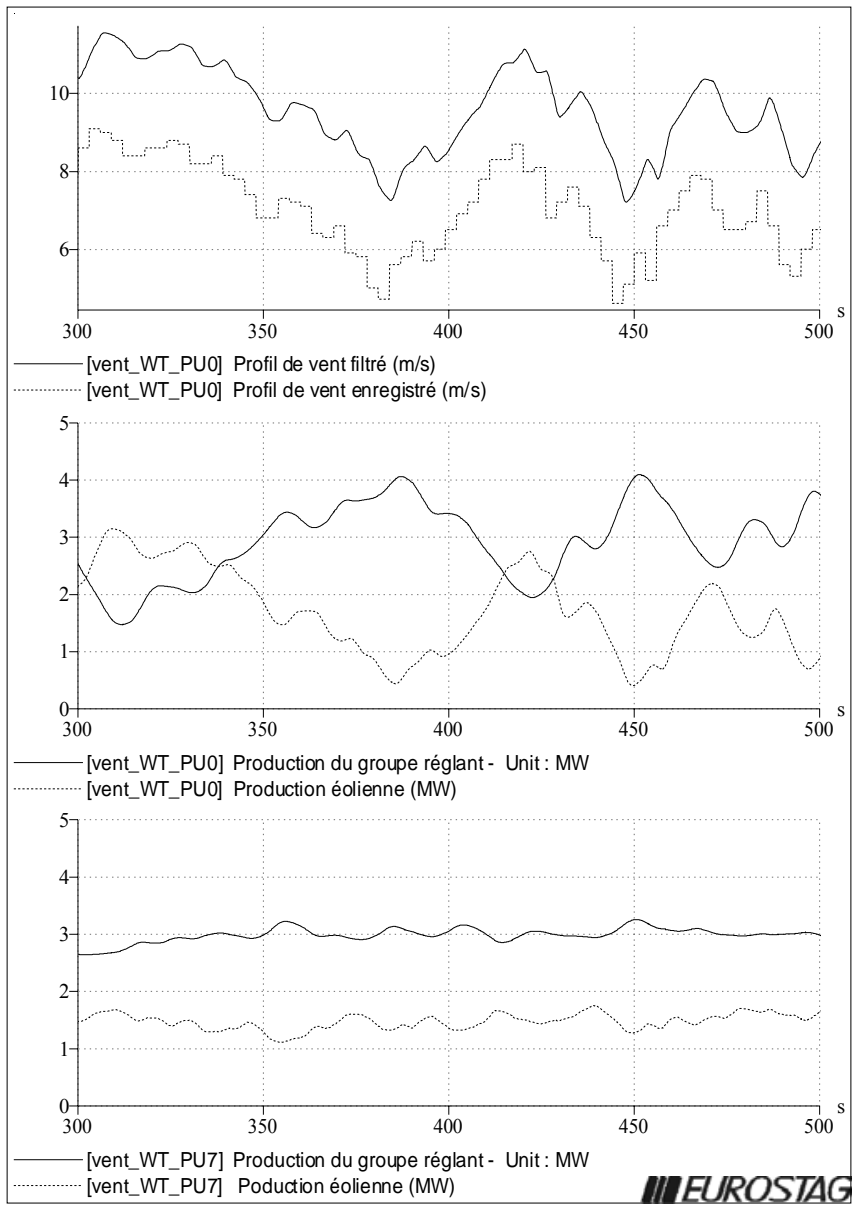

Fig. 24. Réponse du système en présence d'éoliennes soumises à un vent identique ou à des vents décalés dans le temps.

La Fig. 25 montre les conséquences de ces fluctuations de puissances sur les variations de fréquence et de tension dans le système. Le premier tracé montre que le profil de fréquence correspondant à la prise en compte de la non simultanéité est maintenu dans un domaine acceptable $(\Delta \mathrm{f}= \pm 0.5 \mathrm{~Hz})$. Dans le cas d'une seule éolienne équivalente, la variation de fréquence est de l'ordre de $0.75 \mathrm{~Hz}$, soit plus que la variation consécutive au déclenchement d'un groupe. Le deuxième tracé montre l'évolution de la moyenne tension pour laquelle l'amplitude des fluctuations est plus que quadruplée si l'on ne tient pas compte de la non simultanéité.

Dans ces simulations, la puissance générée par la ferme éolienne représente en moyenne un douzième de la puissance consommée, ce qui correspond à un taux de pénétration de $12 \%$.

L'enregistrement présenté en Fig. 26 montre qu'en réalité les variations de puissance sont encore plus réduites en présence d'un grand nombre d'éoliennes présentes dans une ferme et des variations de la vitesse du vent dans le temps et dans l'espace. Ceci est particulièrement perceptible lorsque l'on considère les réponses des 4 composantes de la ferme. 


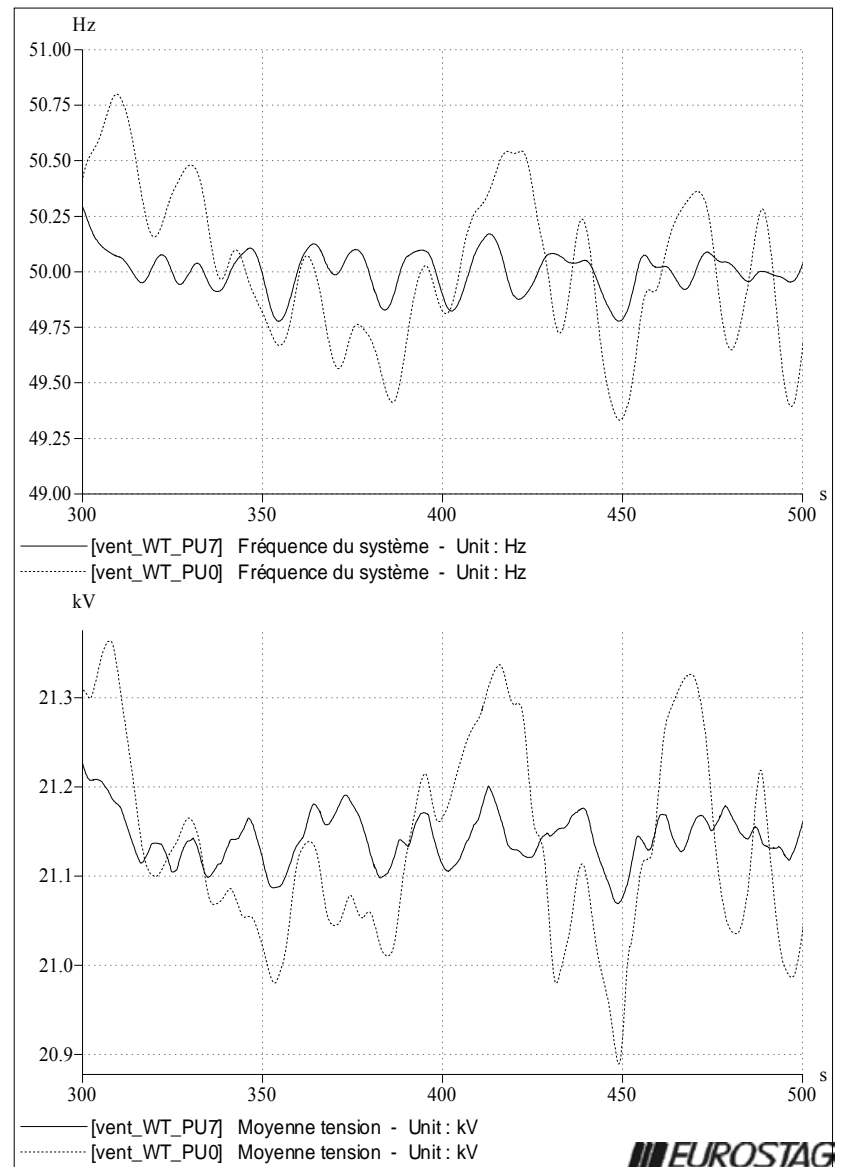

Fig. 25. Variation de fréquence et de tension. Pointillés : éoliennes soumises à un vent identique. Traits continus : éoliennes soumises à des vents décalés dans le temps.

Cela montre que si la production éolienne est considérée comme l'a été jusqu'à présent la charge, c'est-à-dire en tenant compte de sa diversité à l'échelle des réseaux de transport (les réseaux nationaux par exemple), la variation de la production ne devrait pas poser trop de difficultés en termes de réglage primaire. En effet si la production éolienne ne peut être prévue précisément à 24 heures, elle présente une bonne persistance et la production totale sur une aire suffisamment vaste ne changera que très progressivement. Ceci est confirmé par les études du Centre de Recherche Technique de Finlande (VTT) sur l'incidence de l'éolien sur le réseau nordique [22]. Elles montrent que l'éolien serait avant tout amené à jouer un rôle dans le cadre du réglage secondaire, ce qui suppose éventuellement une adaptation de l'architecture du marché pour ne pas inutilement pénaliser l'éolien (celui-ci ne peut évidemment s'inscrire aisément dans le marché du lendemain vu les aléas de la prévision).

Cela étant dit, le fonctionnement actuel est fortement influencé par la non rentabilité du renouvelable en général et de l'éolien en particulier.

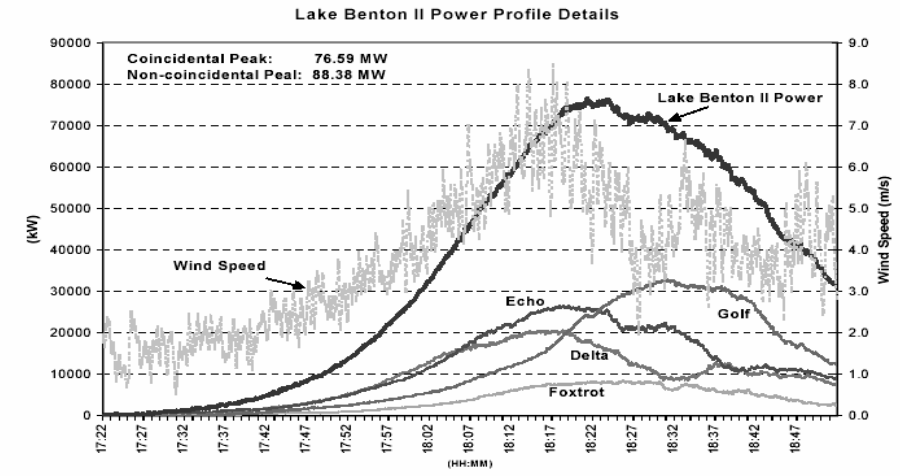

Fig. 26. Production de la ferme du lac Benton [26]

\section{B. Participation au réglage primaire d'une éolienne}

Afin d'illustrer la possibilité pour une éolienne à vitesse variable de participer au réglage primaire de fréquence, le réseau simple de la Fig. 27 a été simulé au moyen du logiciel Simulink. Il comprend une source principale de 3 MVA assurant des réglages primaire et secondaire, une éolienne de $750 \mathrm{~kW}$ basée sur une génératrice à aimants permanents et deux charges identiques ( $\left.P_{c h}=800 \mathrm{~kW} ; Q_{c h}=50 \mathrm{kVAR}\right)$.

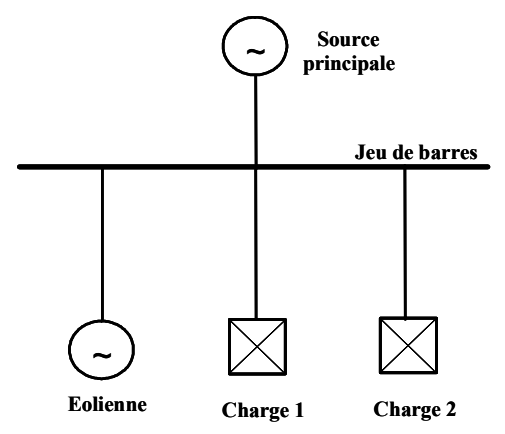

Fig. 27. Configuration du réseau simulé.

Pour participer au réglage primaire de fréquence, la puissance générée par l'éolienne doit être inférieure à la puissance nominale qu'elle pourrait générer afin de disposer d'une réserve primaire d'énergie. Dans l'exemple simulé à la Fig. 28, un vent constant d'une valeur élevée est considéré. Initialement, seule la charge 1 est connectée et l'enclenchement de la charge 2 induit une variation de la fréquence. La puissance générée par l'éolienne et la fréquence du réseau sont présentées pour deux modes de réglages différents de l'éolienne. En trait discontinu, l'éolienne génère la puissance maximale et ne participe pas au réglage primaire. En trait continu, l'éolienne génère une puissance inférieure de $15 \%$ à celle que le vent lui permettrait de fournir et elle participe au réglage primaire suivant une droite de réglage similaire à celle présentée à la Fig. 21. La comparaison des deux courbes d'évolution de la fréquence met en évidence une variation de fréquence sensiblement plus faible lorsque l'éolienne participe au réglage primaire. Il faut toutefois noter que ce résultat est obtenu en considérant un rapport entre la puissance éolienne et la puissance nominale du groupe classique, ainsi qu'un taux de pénétration, élevés. 


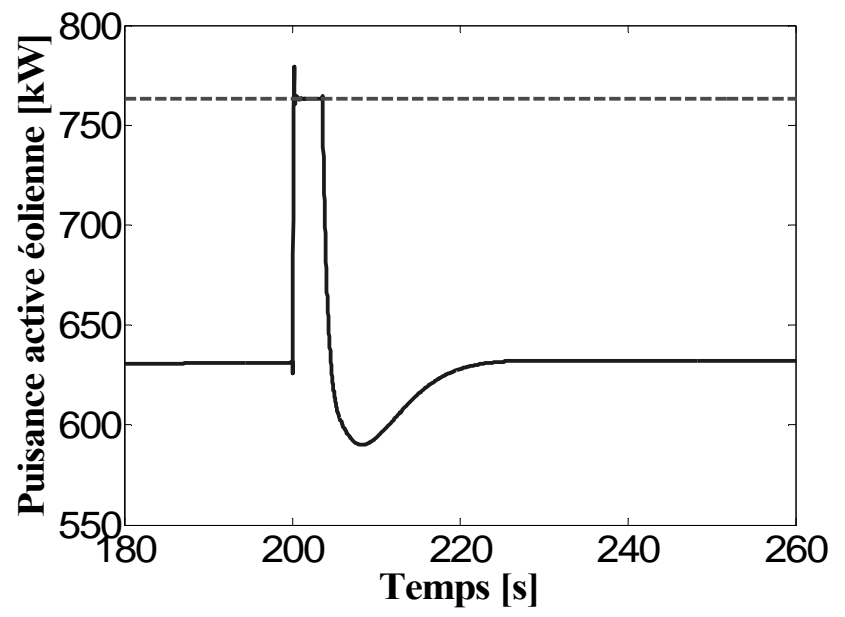

(a)

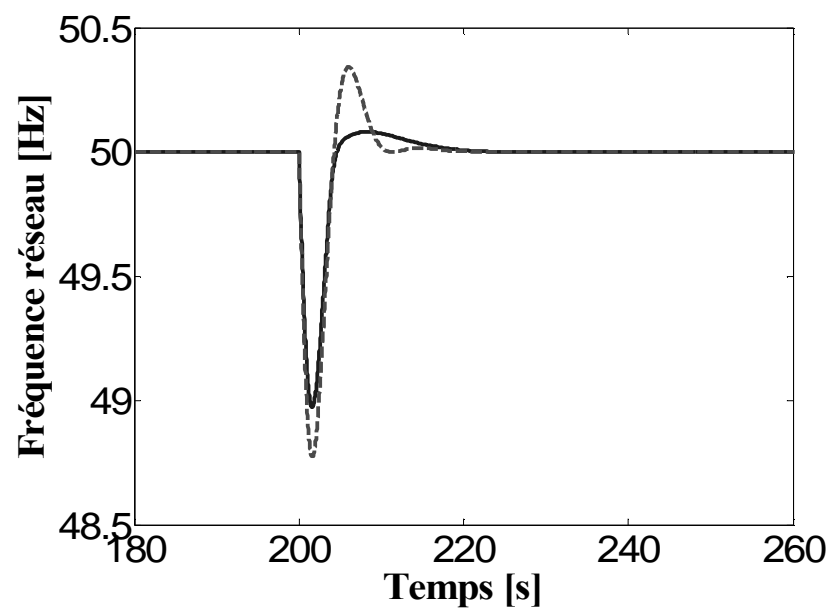

(b)

Fig. 28. Comparaison des modes de réglages de l'éolienne soumise à un vent constant. (a): Puissance active débitée par l'éolienne. (b) : Fréquence du réseau. Traits discontinus: Fonctionnement MPPT. Traits continus : Fonctionnement avec réglage primaire.

La Fig. 29 montre le même type de simulation qu'à la Fig.28, mais en considérant un vent fluctuant. La charge 2 est enclenchée à $t=375 \mathrm{~s}$. Une fonction de lissage est introduite sur la puissance $P_{0}$ (Fig. 21), tout en maintenant une réserve d'environ $15 \%$ de la puissance disponible et une dynamique satisfaisante du réglage primaire. La Fig. 29 montre que 1'on obtient effectivement un certain lissage de la puissance générée par l'éolienne, ainsi qu'une réduction des fluctuations de la fréquence du réseau. Dans l'exemple étudié, la puissance générée par l'éolienne est contrôlée en agissant sur le couple de la génératrice à aimants à attaque directe (sans multiplicateur). Cette méthode induit des fluctuations de vitesse de la turbine afin d'adapter le coefficient de puissance (Fig. 1). Un réglage simple d'orientation des pales intervient pour limiter cette vitesse. Un réglage similaire pourrait être réalisé en agissant uniquement sur l'orientation des pales mais la dynamique du réglage est alors plus lente.

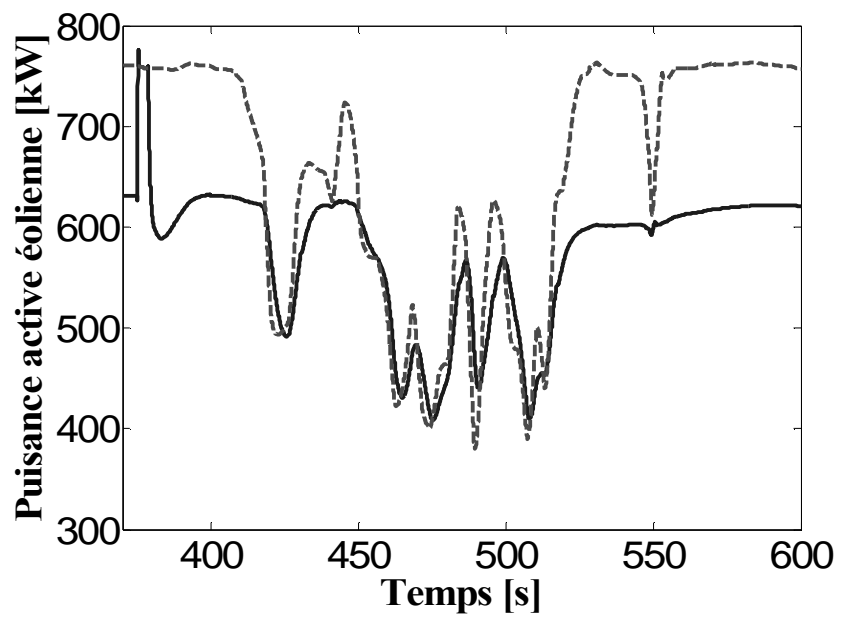

(a)

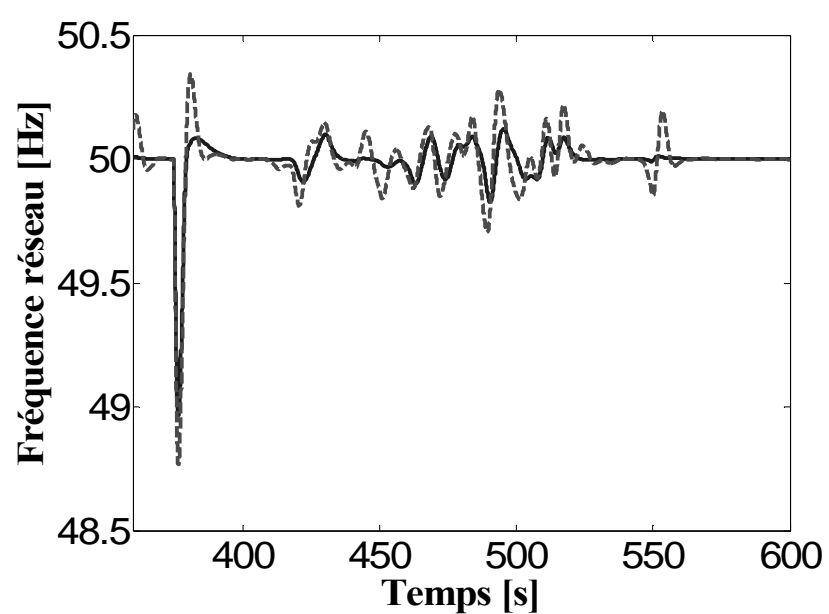

(b)

Fig. 29. Comparaison des modes de réglages de l'éolienne soumise à un vent variable. (a): Puissance active débitée par l'éolienne. (b) : Fréquence du réseau. Traits discontinus: Fonctionnement MPPT. Traits continus : Fonctionnement avec réglage primaire.

\section{VIII.CONCLUSIONS ET PERSPECTIVES}

Parmi les recommandations de l'UCTE [1], en vue d'assurer un développement de l'éolien dans le réseau électrique tout en maintenant sa fiabilité, on peut noter l'accélération des procédures nécessaires à la construction de nouvelles lignes, la définition d'un taux minimum de stabilité imposé aux exploitants de parc éolien par les Etats, le maintien en service à proximité de zones ventées de capacités de production traditionnelles et l'élaboration d'un vaste planning Européen de l'énergie éolienne qui permettrait d'éviter la surconcentration de turbines dans une région, même bien ventée.

Une intégration harmonieuse de l'éolien sera favorisée par :

- Le foisonnement du gisement éolien lissant la puissance totale générée par des fermes éoliennes 
réparties sur un vaste territoire et permettant d'assurer un minimum de production éolienne.

- L'utilisation de l'électronique de puissance dans la liaison au réseau des éoliennes à vitesse variable offrant de nouvelles possibilités de participation au réglage de la fréquence et de la tension, ainsi que le maintien de leur connexion au réseau en présence de creux de tension. Ce sont les éoliennes basées sur une génératrice synchrone à aimants qui sont les plus prometteuses de ce point de vue.

- Le développement du stockage de l'énergie à court et long terme associé aux éoliennes ou aux fermes d'éolienne [27].

- Le développement de systèmes multisources, associant de l'éolien avec d'autres sources aléatoires (par exemple hydraulique) ou conventionnelles, avec une gestion intégrée et optimisée de l'énergie.

Enfin, le développement de l'éolien sera grandement favorisé par une meilleure prévision, de plusieurs heures à plusieurs jours, du potentiel éolien.

\section{REMERCIEMENTS}

Le banc d'essais a été financé par Forclum Ingénierie, Innovelect, HEI, et dans le cadre du Centre National de Recherche Technologique (CNRT) Futurelec par la Région et la DRRT Nord-Pas de Calais, et par le FEDER.

Les informations mesurées sur une éolienne à vitesse variable ont été acquises par Laborelec dans le cadre du programme Futurelec1.

Les études sur la participation au réglage de la fréquence des éoliennes sont réalisées dans le cadre du programme Futurelec6, financés par la Région Nord-Pas de Calais, SuezTractebel, Supélec et HEI.

\section{REFERENCES}

[1] Seven actions for a successful integration of wind power into European electricity system, UCTE, 17 mai 2005, www.ucte.org.

[2] A. Merlin, «Renewables : to what extent can the existing French system cope with dispersed generation? ?, Electra (CIGRE), n² 219, avril 2005, pp. 4 - 11.

[3] T. Ackermann, « Wind power in power systems », Wiley, 2005.

[4] C. Rap, "Variable localement, régulière globalement », Systèmes solaires, ${ }^{\circ} 165,2005$, pp.36-38.

[5] J-Y. Grandidier, "Eolien et hydraulique, des fiançailles réussies », Systèmes solaires, $\mathrm{n}^{\circ} 165,2005$, pp.39-42.

[6] N. Jenkins, R. Allan, P. Crossley, D. Kirschen, G. Strbac, « Embedded generation ", The Institution of Electrical Engineers (IEE), London, 2000 .

[7] M. Crappe, "Commande et régulation des réseaux électriques », Hermès Science, Paris 2003.

[8] Décret du 27 juin 2003 «Prescriptions techniques générales de conception et de fonctionnement auxquelles doivent satisfaire les installations en vue de leur raccordement au réseau public de transport de l'électricité », Ministère de l'économie, des finances et de l'industrie.

[9] Arrêté du 4 juillet 2003 «Prescriptions techniques de conception et de fonctionnement pour le raccordement au réseau public de transport d'une installation de production d'énergie électrique ", Ministère de l'économie, des finances et de l'industrie.

[10] B. Multon, X.Roboam, B.Dakyo, C.Nichita, O.Gergaud, H.Ben Ahmed, "Aérogénérateurs électriques », Techniques de l'ingénieur, D3960, 2004.

[11] N. Laverdure, D. Roye, S.Bacha, R. Belhomme, «Technologie des systèmes éoliens - Intégration dans les réseaux électriques », Revue 3EI, n³9, décembre 2004, pp. 14-25.

[12] B. Paluch, «Introduction à l'énergie éolienne », REE, n5, mai 2005, pp. 35-50.

[13] B. Francois, B. Robyns, E. De Jaeger, F. Minne, «Technologies d'éolienne de forte puissance connectée au réseau de moyenne tension », REE, n5, mai 2005, pp 65-74.

[14] S. El Aimani, B. François, B. Robyns, F. Minne, «Modeling and Simulation of Doubly Fed Induction Generators for Variable Speed Wind Turbines integrated in a Distribution Network », Proceedings of the $10^{\mathrm{h}}$ European Conference on Power Electronics and Applications : EPE'2003, September 2 - 4, 2003, Toulouse (France), CD, ISBN 9075815-07-7.

[15] C. Saudemont, L. Leclercq, B. Robyns, G. Cimuca, M. Radulescu, « Développement d'un émulateur temps réel d'un système de génération éolienne associé à un stockage d'énergie », REE, n¹1, décembre 2004, pp. 49-59.

[16] C. Saudemont, G. Cimuca, B. Robyns, M. Radulescu, « Grid connected or stand-alone real-time variable speed wind generator emulator associated to a flywheel energy storage system $"$, Proceedings of the $11^{\text {th }}$ Conference EPE 2005, Dresde, 11 - 14 septembre 2005.

[17] P. Bartholomeus, P. Lemoigne, C. Rombaut, «Etude des limitations en puissance des convertisseurs et apport des techniques multiniveaux ", Actes du colloque Electronique de puissance du Futur, EPF'96, Grenoble, 1996, pp. $121-126$

[18] P. Vercauteren «C-Power NV : Le parc éolien offshore en Belgique », Actes de la journée d'étude SRBE-SEE sur le thème «Eolien et réseaux : enjeux », Lille, 22 mars 2005.

[19] J. - L. Fraisse, « Le raccordement de la production décentralisée en HTA et BT », Revue REE, n7, Juillet 2002.

[20] B. Robyns, P. Bastard, «Production décentralisée d'électricité : contexte et enjeux techniques », Revue 3EI, n³9, décembre 2004, pp. 5-13.

[21] Mémento de la sureté du système électrique, RTE, Edition 2004, ISBN $-\mathrm{n}^{\circ} 2-912440-13-0$, pp. $32-41$, www.rte-france.com.

[22] Hannele Holttinen, « The impact of large scale wind power production on the Nordic electrical system », VTT Processes, Finlande, Décembre 2004.

[23] «FINAL REPORT of the Investigation Committee on the 28 September 2003 Blackout in Italy », UCTE, avril 2004, www.ucte.org.

[24] S. Chatellier, P. Juston, "Intégration de l'éolien dans le système électrique. Etude de fonctionnement dynamique sur creux de tension. », Actes de la journée d'étude SRBE-SEE sur le thème «Eolien et réseaux : enjeux », Lille, 22 mars 2005.

[25] E. F. Mogos, X. Guillaud, V. Rogez, «A voltage regulation system for pitch-controlled variable-speed wind turbine », Proceedings of the 2 th International Conference on Securing Critical Infrastructures, CRIS 2004, Grenoble, 25- 27 octobre 2004.

[26] «Wind Farm Power Fluctuations, Ancillary Services, and System Operating Impact Analysis », NREL/CP - 500 - 30547.

[27] B. Robyns, A. Ansel, A. Davigny, C. Saudemont, G. Cimuca, M. Radulescu, J-M. Grave « Apport du stockage de l'énergie à l'intégration des éoliennes dans les réseaux électriques. Contribution aux services système », Revue de l'Electricité et de l'Electronique (REE), n ${ }^{\circ} 5$, mai 2005 .

\section{BIOGRAPHIES}

Benoît Robyns est né à Bruxelles, Belgique, en 1963. Il est diplômé "Ingénieur Civil Electricien" et "Docteur en Sciences appliquées" de l'Université Catholique de Louvain (UCL), Belgique, respectivement en 1987 et 1993. En 2000, il reçoit le diplôme d' "Habilitation à Diriger des Recherches" de l'Université des Sciences et Technologies de Lille (USTL). Il est responsable du Département Génie Electrique de l'Ecole des Hautes 
Etudes d'Ingénieur (HEI) de Lille. Il est aussi responsable de l'équipe Réseaux Electriques et Systèmes Energétiques (RESE) du Laboratoire d'Electrotechnique et d'Electronique de Puissance (L2EP) de Lille.

Arnaud Davigny est né à Lens, France, en 1972. Il obtient en 1998 le «Diplôme d'Etudes Approfondies de génie électrique » de l'Université des Sciences et Technologies de Lille (USTL), France. Il intègre le département Génie Electrique de l'Ecole des Hautes Etudes d'Ingénieur (HEI) de Lille, en 2001. Depuis septembre 2003, il est doctorant au sein de l'équipe RESE (Réseaux Electriques et Systèmes Energétiques) du Laboratoire d'Electrotechnique et d'Electronique de Puissance (L2EP) de Lille. Son travail de thèse concerne la participation aux services systèmes de fermes d'éoliennes à vitesse variable associant du stockage inertiel d'énergie et se déroule sous la direction du Professeur B. Robyns. Il est membre de la «Société des Electriciens et des Electroniciens» (SEE) et de la «Société Française d'Energie Nucléaire » (SFEN).

Christophe Saudemont est né en à Douai, France, en 1971. Il est diplômé du Doctorat de Génie Electrique de l'Université des Sciences et Technologies de Lille (USTL) en 1999. Il intègre le département de Génie Electrique de l'Ecole des Hautes Etudes d'Ingénieur (HEI) de Lille, France, en 2001. Depuis 2002, il est Chercheur au sein du Laboratoire d'Electrotechnique et d'Electronique de Puissance (L2EP) de Lille, au sein de l'équipe RESE (Réseaux Electriques et Systèmes Energétiques), au sein de laquelle ses travaux portent sur les énergies renouvelables, la production décentralisée d'électricité, et la connexion de ces sources au réseau électrique. Il est membre de la "Société française des Electriciens et des Electroniciens" (SEE).

Aymeric Ansel est né à Amiens, France, en 1974. Il est diplômé Ingénieur de l'Ecole Supérieure d'Ingénieurs en Electrotechnique et Electronique d'Amiens (ESIEE-Amiens) en 1997. En 2001, il reçoit le grade de "Docteur en Génie Electrique" de l'Université d'Artois, Arras, France. Depuis Septembre 2001, Aymeric Ansel est Enseignant-Chercheur à l'Ecole des Hautes Etudes d'Ingénieur (HEI) de Lille, France, et Chercheur au sein du Laboratoire d'Electrotechnique et d'Electronique de Puissance (L2EP) de Lille. Ses domaines d'intérêt sont la conception et la modélisation des machines électriques ainsi que, depuis 2001, les énergies renouvelables et la production décentralisée d'électricité. Il est membre de la "Société française des Electriciens et des Electroniciens" (SEE).

Vincent Courtecuisse est né à Lesquin, France, en 1980. Il obtient en 2005 le «Master de Recherche en Energie Electrique et Développement Durable » de 1'Université des Sciences et Technologies de Lille (USTL), France. Il est actuellement en première année de thèse au sein au sein de l'équipe RESE (Réseaux Electriques et Systèmes Energétiques) du Laboratoire d'Electrotechnique et d'Electronique de Puissance (L2EP) de Lille. Ses travaux de recherche portent sur l'intégration de centrales multisources incluant de l'éolien dans les réseaux électriques.

Bruno Francois a obtenu son doctorat de génie électrique de l'Université des Sciences et Technologies de Lille en 1996 et son "Habilitation à Diriger des Recherches" en 2003. Il travaille au sein de l'Ecole Centrale de Lille comme enseignant et en tant que chercheur au sein de l'équipe Réseaux Electriques et Systèmes Energétiques (RESE) du Laboratoire d'Electrotechnique et d'Electronique de Puissance (L2EP) de Lille et au sein du Centre National de Recherche Technologique "Machines et Réseaux Electriques du Futur". Ses thématiques de recherche sont centrées sur la modélisation des systèmes et la conception de leur commande, l'électronique de puissance dans les réseaux électriques, la fourniture de "services système" par la production distribuée, la supervision de productions distribuées au sein des micro réseaux.
Sophie Plumel est professeur à Supélec depuis 2002. Elle a obtenu un diplôme d'ingénieur de l'ENSEEIHT en Electrotechnique en 1993. Elle est agrégée de Génie Electrique et a enseigné en lycée de 1995 à 1999. Elle a obtenu le doctorat de l'Université Pierre et Marie Curie (Paris 6) en 2002. Ses activités de recherche portent sur le fonctionnement et la planification des réseaux de transport d'électricité en environnement concurrentiel.

Jacques Deuse est né en Belgique, le 25 mars 1949. Diplômé Ingénieur Electricien \& Mécanicien en 1972 de l'Université de Liège, Belgique, il a reçu le degré de Docteur en Sciences Appliquées en 1976 de cette même Université. De 1972 à 1976 il a travaillé comme assistant à l'Institut Montefiore. In 1977, il intègre le Département de planification de la société Intercom (devenue depuis Electrabel) en tant qu'expert en système électrique. En 1989, il rejoint Tractebel Engineering, où il est actuellement Ingénieur en Chef, dans le service « Power System Consulting » du Département «Energy \& Industrial Solutions. » 